\title{
Well-posedness for a mean field model of Ginzburg-Landau vortices with opposite degrees
}

\author{
Edoardo Mainini
}

\begin{abstract}
In this paper we analyze the hydrodynamic equations for Ginzburg-Landau vortices as derived by E (Phys. Rev. B. 50(3):1126$1135,1994)$. In particular, we are interested in the mean field model describing the evolution of two patches of vortices with equal and opposite degrees. Many results are already available for the case of a single density of vortices with uniform degree. This model does not take into account the vortex annihilation, hence it can also be seen as a particular instance of the signed measures system obtained in Ambrosio et al. (Ann. Inst. H. Poincaré Anal. Non Linéaire 28(2):217246, 2011) and related to the Chapman et al. (Eur. J. Appl. Math. $7(2): 97-111,1996)$ formulation. We establish global existence of $L^{p}$ solutions, exploiting some optimal transport techniques introduced in this context in Ambrosio and Serfaty (Commun. Pure Appl. Math. LXI(11):1495-1539, 2008). We prove uniqueness for $L^{\infty}$ solutions, as expected by analogy with the incompressible Euler equations in fluidodynamics. We also consider the corresponding Dirichlet problem in a bounded domain. Moreover, we show some simple examples of 1-dimensional dynamic.
\end{abstract}

Mathematics subject classification (2010). 35M, 35Q35, 37C10, 49K20.

Keywords. Superconductivity, Hydrodynamic equations, Vortex density, Vortex degree, Wasserstein distance, Optimal transportation.

\section{Introduction}

Let $\Omega \subseteq \mathbb{R}^{2}$ be a smooth open domain. Let $\chi_{\Omega}$ denote its indicator function. We are going to study the following model for Ginzburg-Landau vortices motion, derived by $\mathrm{E}$ in [10]: 


$$
\left\{\begin{array}{l}
\frac{d}{d t} \mu_{1}(t)-\operatorname{div}\left(\chi_{\Omega} \nabla h_{\mu(t)} \mu_{1}(t)\right)=0 \\
\frac{d}{d t} \mu_{2}(t)+\operatorname{div}\left(\chi_{\Omega} \nabla h_{\mu(t)} \mu_{2}(t)\right)=0 \\
\mu(t)=\mu_{1}(t)-\mu_{2}(t),
\end{array}\right.
$$

with the initial data $\mu_{1}(0)=\mu_{1}^{0}$ and $\mu_{2}(0)=\mu_{2}^{0}$. Here $\Omega$ represents the section of the superconducting sample, while $\mu_{1}$ (resp. $\mu_{2}$ ) represents the density of vortices with positive (resp. negative) topological degree. We are dealing with a coupled system of evolution equations, having the form of continuity equations. The corresponding velocity vector fields are equal and opposite, and written in terms of a function $h_{\mu(t)}$, which is solution, for any $t$, to

$$
\begin{cases}-\Delta h_{\mu(t)}=\mu(t) & \text { in } \Omega \\ h_{\mu(t)}=0 & \text { on } \partial \Omega .\end{cases}
$$

In order to address the possible non conservation of mass in the domain, we deal with measure solutions and admit concentration on the boundary. We also allow for the case $\Omega=\mathbb{R}^{2}$, with the same elliptic equation in (1.2) satisfied by $h_{\mu}$, omitting the boundary condition: in such case, we are considering the solution given by convolution with the logarithmic Green kernel corresponding to the Laplace operator in the whole plane. For $\alpha \geq 0$, let us denote by $\mathcal{M}_{\alpha}^{2}(\bar{\Omega})$ the set of positive measures over $\bar{\Omega}$ with finite second moment and total mass equal to $\alpha$. The basic problem could be formulated as follows: given an initial datum

$$
\left(\mu_{1}^{0}, \mu_{2}^{0}\right) \in \mathcal{M}_{\alpha}^{2}(\bar{\Omega}) \times \mathcal{M}_{\beta}^{2}(\bar{\Omega}), \quad \chi_{\Omega}\left(\mu_{1}^{0}-\mu_{2}^{0}\right) \in H^{-1}(\Omega), \quad \alpha, \beta \geq 0,
$$

find a couple of measures $\left(\mu_{1}(t), \mu_{2}(t)\right)$ in the same space which is solution to (1.1)-(1.2). Here the $H^{-1}$ condition is the natural one coming from the elliptic problem. Actually, in this paper we are going to consider initial data with $L^{p}$ regularity in the interior of the domain, and to show that such regularity is conserved in the dynamic. Our aim is to establish a satisfying well-posedness picture for system (1.1)-(1.2). We will prove a global existence result, as well as a uniqueness result (global if $\Omega=\mathbb{R}^{2}$ ). We will construct solutions as gradient flows of the energy functional

$$
\left(\mu_{1}, \mu_{2}\right) \mapsto \Phi(\mu):=\frac{1}{2} \int_{\Omega} h_{\mu} d \mu,
$$

where $\mu=\mu_{1}-\mu_{2}$ (the energy depends only on the difference of $\mu_{1}$ and $\mu_{2}$ ).

Let us state the main theorems.

Theorem 1.1. Let $p \geq 4$. Let $\left(\mu_{1}^{0}, \mu_{2}^{0}\right) \in \mathcal{M}_{\alpha}^{2}(\bar{\Omega}) \times \mathcal{M}_{\beta}^{2}(\bar{\Omega})$ be such that $\left(\chi_{\Omega} \mu_{1}^{0}, \chi_{\Omega} \mu_{2}^{0}\right)$ belongs to $L^{p}(\Omega)^{2}$. There exists a weakly continuous map $t \in$ $[0,+\infty) \mapsto\left(\mu_{1}(t), \mu_{2}(t)\right) \in \mathcal{M}_{\alpha}^{2}(\bar{\Omega}) \times \mathcal{M}_{\beta}^{2}(\bar{\Omega})$ such that:

$$
\left\|\chi_{\Omega} \mu_{1}(t)\right\|_{p}+\left\|\chi_{\Omega} \mu_{2}(t)\right\|_{p} \leq K_{p}
$$


where $K_{p}$ is a constant depending only on the initial data, $\mu_{1}(0)=\mu_{1}^{0}$, $\mu_{2}(0)=\mu_{2}^{0}$ and there hold

$$
\begin{aligned}
& \frac{d}{d t} \mu_{1}(t)-\operatorname{div}\left(\chi_{\Omega} \nabla h_{\mu(t)} \mu_{1}(t)\right)=0 \quad \text { in } \mathcal{D}^{\prime}\left((0,+\infty) \times \mathbb{R}^{2}\right), \\
& \frac{d}{d t} \mu_{2}(t)+\operatorname{div}\left(\chi_{\Omega} \nabla h_{\mu(t)} \mu_{2}(t)\right)=0 \quad \text { in } \mathcal{D}^{\prime}\left((0,+\infty) \times \mathbb{R}^{2}\right) .
\end{aligned}
$$

Here $\mu(t)=\mu_{1}(t)-\mu_{2}(t)$ and $h_{\mu(t)}$ is defined through (1.2). Moreover, the maps $t \mapsto \mu_{1}(t)(\Omega), t \mapsto \mu_{2}(t)(\Omega)$ are nonincreasing, $\Phi\left(\mu_{1}^{0}-\mu_{2}^{0}\right)<+\infty$ and one has the energy dissipation equality

$$
\Phi(\mu(t))+\int_{s}^{t} \int_{\Omega}\left|\nabla h_{\mu(r)}\right|^{2} d\left(\mu_{1}(r)+\mu_{2}(r)\right) d r=\Phi(\mu(s)), \quad t \geq s \geq 0
$$

Before passing to the uniqueness result we remark the fact that, having measures which are $L^{p}$ in the interior of the domain and not only $H^{-1}$, we avoid complications in the definition of the solution. Indeed, as we will specify later in Sect. 4 , in our case by elliptic regularity $\nabla h_{\mu}$ is a continuous function, so that the products $\nabla h_{\mu} \mu_{1}, \nabla h_{\mu} \mu_{2}$ are well defined and we can work with the standard weak formulation of Theorem 1.1. In this paper we do not deal with the the general $H^{-1}$ case, for which one should introduce a generalized formulation as done in [3].

Theorem 1.2. Let $p=+\infty$. Let $\left(\mu_{1}^{0}, \mu_{2}^{0}\right)$ belong to $L^{\infty}(\Omega)^{2}$ and be supported in $\Omega$. Then there exists $T>0$ such that $\operatorname{supp} \mu_{1}(t) \subset \Omega, \operatorname{supp} \mu_{2}(t) \subset \Omega$ for any $t<T$ and the solution given by Theorem 1.1 is the unique solution to (1.1), starting from $\left(\mu_{1}^{0}, \mu_{2}^{0}\right)$, such that $\left(\mu_{1}(t), \mu_{2}(t)\right) \in L^{\infty}\left((0, T) ; L^{\infty}(\Omega)^{2}\right)$. If $\Omega=\mathbb{R}^{2}$, we may let $T=+\infty$.

\section{Plan of the paper}

In Sect. 2 we discuss the physical framework in which (1.1)-(1.2) is derived and some results for related models. In Sect. 3 we give some explicit solutions and analyze the behavior of (1.1)-(1.2) in one space dimension. Sections 4 and 5 are devoted respectively to prove Theorem 1.1 and Theorem 1.2.

\section{The model}

The Chapman-Rubinstein-Schatzman model. The equations we are dealing with come from the vortex motion analysis in superconductors. It is well known that, at low temperatures, a (type-II) superconductor subject to an external magnetic field presents different behaviors. In the so called mixed phase, the superconducting region in the sample is confined outside the vortices. Each vortex corresponds to a zero of the order-parameter complex function appearing in the Ginzburg-Landau energy, and carries a signed topological degree, see also [23] and the references therein. In the mixed state, it is observed that the vortex reticular structure can melt and form a liquid state. Then, it is interesting to study the motion of such a vortex liquid. In [6], Chapman, 
Rubinstein and Schatzman introduce the following mean field model, similar to (1.1)-(1.2), for the evolution of superconducting vortices (see also [5]):

$$
\frac{d}{d t} \mu(t)-\operatorname{div}\left(\nabla h_{\mu(t)}|\mu(t)|\right)=0 \quad \text { in } \Omega,
$$

the coupling being

$$
\begin{cases}-\Delta h_{\mu}+h_{\mu}=\mu & \text { in } \Omega \\ h_{\mu}=1 & \text { on } \partial \Omega .\end{cases}
$$

This can be considered as the reference physical model. Here, $h$ represents the intensity of the magnetic field induced in the superconducting sample, whose section is $\Omega$, whereas $\mu$ is the (macroscopic) vortex density. Since the vortices may possess degrees of different sign, the density $\mu$ is signed. In particular, it is suitable to assume that the vortices have degree 1 or -1 , since vortices with different degrees are not stable (see [5] or [10]).

Previous studies of the model. Different studies have been devoted to this model, both from the analytical and the numerical point of view, mostly in the case of positive density $\mu$. We refer for instance to $[8,11,16,20,24]$. In particular, the interpretation as gradient flow in the space of probability measures has been introduced in [3]. It consists in viewing the solution as a curve of maximal slope for a suitable energy functional (related to the Ginzburg-Landau functional). The curve can be constructed by a discrete scheme (known as the minimizing movement scheme) in the probability space, endowed with the optimal transport structure. In Sect. 4 we will very briefly recall the main elements of the theory. In $[2,17]$, as well as in this paper, we continue the analysis in this framework.

For positive solutions in the whole plane, the model reduces to a single equation of (1.1) (this happens when the vortex degree is uniform, say equal to 1 , and also one recovers the model of $\mathrm{E}$ in this case). The difference lies in the elliptic equation, but it is not a substantial difference. Hence, even for equation (2.1), we could consider the basic coupling given by $-\Delta h_{\mu}=\mu$, as done in [20] and in [2, Sect. 6]. In this case we are left with the evolution model

$$
\frac{d}{d t} \mu_{1}(t)-\operatorname{div}\left(\left(\nabla \Delta^{-1} \mu_{1}(t)\right) \mu_{1}(t)\right)=0
$$

where $\mu_{1}$ is the positive density of vortices. Equation (2.3) has been studied in the already mentioned papers: existence results have been obtained through different approximation schemes. In general we have existence of (positive) solutions (both measures and $L^{p}$ solutions), and uniqueness for $L^{\infty}$ solutions.

Presence of the boundary. We draw the attention to some more features of the physical model. In general it is interesting to study the case of a bounded domain $\Omega$, describing the material sample, and to take into account the possibility that some mass enters or exits the domain. We can describe the phenomenon letting the measure $\mu$ concentrate on the boundary. Instead of the natural Neumann condition, in these cases we have Dirichlet conditions on $\partial \Omega$ for the elliptic problem. The presence of the boundary makes well-posedness 
results more difficult. In [3], the authors pointed out that the right formulation in this case should have a zero velocity field on the boundary. This explains the appearance of the factor $\chi_{\Omega}$ also in (1.1). Concerning positive solutions in presence of boundary, [3] contains an existence result of measures and $L^{p}$ solutions and a short time uniqueness result for $L^{\infty}$ solutions. Even if the possible concentration of mass on the boundary seems to prevent the corresponding global uniqueness in time, this problem can also be tackled by introducing a more precise condition. Indeed, it is shown in [17] that, at least for a convex domain $\Omega$, there is existence and uniqueness of solutions with $L^{\infty}$ interior part and such that

$$
\left\langle\nabla h_{\mu(t)}(x), y-x\right\rangle \geq 0 \quad \text { for all }(x, y) \in \operatorname{supp}\left(\chi_{\partial \Omega} \mu(t)\right) \times \bar{\Omega},
$$

for any $t>0$. This means that the (limit of the) velocity field on the boundary, whenever some mass is there, is directed outside the domain.

The general signed case and open problems. The most relevant fact, for what concerns our analysis, is the charged nature of vortices. Indeed, we stress that the full model (2.1) is concerned with a signed density $\mu$. Many problems are open in the signed case. An existence and uniqueness result for Sobolev or $C^{\alpha}$ solutions has been established in [20]. In [2] an attempt of generalizing the full results of [3] is made, and it turns out that there exist $L^{p}$ solutions (possibly with a boundary part in addition) to the system

$$
\left\{\begin{array}{l}
\frac{d}{d t} \mu_{1}(t)-\operatorname{div}\left(\chi_{\Omega} \nabla h_{\mu(t)} \mu_{1}(t)\right)=-\sigma_{\mu}(t), \\
\frac{d}{d t} \mu_{2}(t)+\operatorname{div}\left(\chi_{\Omega} \nabla h_{\mu(t)} \mu_{2}(t)\right)=-\sigma_{\mu}(t), \\
\mu(t)=\mu_{1}(t)-\mu_{2}(t) .
\end{array}\right.
$$

where $\sigma_{\mu}$ is a positive measure representing a mass sink. The presence of such a term is quite natural, since we expect that vortices with opposite degrees attract each other, and may cancel during the evolution. Actually, here $\mu_{1}$ and $\mu_{2}$ are not the positive and negative parts of $\mu(t)$, but simply two positive measures which may overlap, and whose difference is $\mu(t)$. The problem of finding solutions to (2.1) is then equivalent to find orthogonality-preserving solutions for system (2.5) $\left(\mu_{1}\right.$ and $\mu_{2}$ are orthogonal measures if their minimum $\left.\mu_{1} \wedge \mu_{2}=0\right)$. This problem seems to be very difficult, also because in the derivation of [2] the term $\sigma_{\mu}$ is highly undetermined.

The E Model. In this paper we will analyze the related model (1.1)-(1.2), which has been introduced by $\mathrm{E}$ in [10]. We will show that the strongest existence and uniqueness results available for the positive case (Eq. (2.3) above) extend to this signed density problem. The model is derived considering the hydrodynamic limit of Ginzburg-Landau equations (in the case of the whole plane). In the formulation of [10] the associated elliptic equation is $-\Delta h_{\mu}+$ $h_{\mu}=\mu$. Here we are considering the simplified, basic model obtained therein in the case of absence of magnetic fields, the elliptic coupling being reduced to $-\Delta h_{\mu}=\mu$ as in (1.2) (see also [9]). In this case $h_{\mu}$ is simply a potential 
generated by the vortices (not the induced magnetic field). We stress again that this way we keep all the mathematical features of the problem.

Notice that (1.1) is also a particular instance of (2.5). In (1.1) we have no mass annihilation terms. In general, we could not expect the orthogonality of initial data to be preserved in this case, as we will also see in Sect. 3. But the interaction between the two parts is similar, because the two measures tend to stop when they overlap (due to the coupled nature of the velocity vector fields), even if they are not cancelling each other. Considering separately the evolution of the positive and negative parts, we are left with an half-way model between the one for the positive case and the one in (2.5). Of course, if we start with distant initial data $\mu_{1}^{0}$ and $\mu_{2}^{0}$, since the velocity of evolution is finite (if the data are regular enough, as remarked later in Sect. 5), we keep orthogonality for some time. Hence, in this case our results give also short time existence and $L^{\infty}$ uniqueness for (2.1). However, the general existence of $L^{p}$ solutions to (2.1) (and the correspondence with (2.5)) is still a major open problem.

Analogy with fluid dynamics. We end this section by pointing out the relation between the E model and the standard incompressible Euler equations for fluid dynamics in vorticity formulation. The analogy is discussed in [10], where the incompressible Euler equations are deduced as the hydrodynamic limit of a nonlinear Schrödinger equation (more precisely the Gross-Pitaevskii equation). In this case it turns out that the velocity vector field is $\nabla^{\perp} \Delta^{-1} \mu$, whereas in (2.3) it is $\nabla \Delta^{-1} \mu$. This rotation makes Eq. (2.3) dissipative. The same velocity field $\nabla^{\perp} \Delta^{-1} \mu$ governs the motion of vortices in superfluids, for instance subject to an external momentum, instead of a magnetic field (see $[22])$.

For the Euler equations in the plane, the standard result of Yudovich entails existence and uniqueness of $L^{\infty}$ solutions (see [27]). Loeper (see [15]) proves the same uniqueness result in the optimal transport setting. We will adopt the same techniques, which are suitable for the coupled system with two densities, in Sect. 5 .

\section{Examples and heuristics}

In this section we collect some examples of explicit solutions for the one dimensional case. To begin with, we consider a single, non coupled equation from system (1.1), that is we consider the problem

$$
\left\{\begin{array}{l}
\partial_{t} \rho-\operatorname{div}\left(\nabla h_{\rho} \rho\right)=0, \\
-\Delta h_{\rho}=\rho \\
\rho(0, x)=\rho^{0}(x) .
\end{array}\right.
$$

We are thinking to positive solutions: in this framework, well-posedness results for the single equation are already available, as observed in the previous section. We refer in particular to $[8,24]$ for some studies about one dimensional models. Notice that in a smooth framework this equation could be written as 
$\partial_{t} \rho-\nabla h_{\rho} \cdot \nabla \rho+\rho^{2}=0$. Hence we have an advection-reaction equation, where the solution decays, due to the sign of the reaction term. Since we consider the one dimensional case, the problem takes the form

$$
\left\{\begin{array}{l}
\partial_{t} \rho-\left(h_{\rho}^{\prime} \rho\right)^{\prime}=0, \\
\rho(0, x)=\rho^{0}(x),
\end{array}\right.
$$

where the prime denotes spatial derivative. Here $h_{\rho}^{\prime \prime}=-\rho$ is the equation for the velocity vector field: let

$$
h_{\rho}^{\prime}=-\int_{0}^{x} \rho(y) d y .
$$

Now let us consider the initial datum $\rho^{0}(x)=\chi_{[-1,1]}(x)$. It is not difficult to check that a bounded solution to $(3.2)-(3.3)$ is

$$
\rho(t, x)=\frac{1}{1+t} \chi_{[-1-t, 1+t]}(x) .
$$

In fact, it is readily seen that $h_{\rho}^{\prime}$, which is continuous, is given by

$$
\chi_{(-\infty,-1-t)}(x)-\frac{x}{1+t} \chi_{[-1-t, 1+t]}(x)-\chi_{(1+t,+\infty)}(x),
$$

so that

$$
h_{\rho}^{\prime}(t, x) \rho(t, x)= \begin{cases}0 & \text { for } x<-1-t, \\ -\frac{x}{(1+t)^{2}} & \text { for }-1-t<x<1+t, \\ 0 & \text { for } x>1+t .\end{cases}
$$

The discontinuity set for the solution is $\{x= \pm(1+t)\}$, and it is immediate to verify that the standard Rankine-Hugoniot condition (see for instance [12]) is satisfied. The behavior of the computed solution is clear: the initial mass supported in the set $[-1,1]$ spreads over the real line. This is of course expected for positive solutions of our equations. Mind that this is also a solution for the mean field model (2.1)-(1.2), since we are in the positive case. See also the numerical results described in [11]. Moreover, such solution is also the unique bounded solution, as a byproduct of the analysis of $[2,3]$, and, of course, as a particular case of the result in the last section of this paper.

If the sign in the Poisson problem $-\Delta h_{\rho}=\rho$ is changed, the sign of the term $\rho^{2}$ also changes, so that it is no more an extinction term. In this case the Rankine-Hugoniot condition shows that the slope of the discontinuity lines in the $x-t$ plane is

$$
\frac{\left[h_{\rho}^{\prime} \rho\right]}{[\rho]}=\frac{ \pm 1 /(1-t)}{1 /(1-t)}= \pm 1,
$$

where [.] denotes the jump. The discontinuity set is then $\{x= \pm(1-t)\}$ and the explicit solution is given by

$$
\rho(t, x)=\frac{1}{1-t} \chi_{[t-1,1-t]}(x), \quad t<1 .
$$

Hence we have divergence in finite time for a bounded initial datum. Since in (1.1) the velocity vector fields are changing sign, one could expect that such 
behavior is possible also for that coupled problem. We will however show in Sect. 4 that global bounded solutions always exist.

Let us now directly investigate system (1.1). We let $\rho_{1}, \rho_{2}$ be the variables. First of all, if $\rho_{1}^{0}=0$ (resp. $\rho_{2}^{0}=0$ ), then the unique solution of the first (resp. the second) equation therein is the null one. In this case we can see that we reduce to study a single, extinction equation of the form (3.1). Another trivial case is $\rho_{1}^{0}=\rho_{2}^{0}$, the system admitting the solution $\rho_{i}(t) \equiv \rho_{i}^{0}, i=1,2$. In general we don't expect to easily obtain explicit solutions as before, but we can argue some qualitative behaviors for a couple of meaningful examples.

Let us fix the initial datum $\rho_{1}^{0}(x)=2 \rho_{2}^{0}(x)=2 \chi_{[0,1]}(x)$. In this case, the initial value of $h_{\rho}^{\prime}$ (as usual we let $\rho:=\rho_{1}-\rho_{2}$ ) is

$$
\begin{cases}0 & \text { for } x<0 \\ -x & \text { for } 0 \leq x \leq 1 \\ -1 & \text { for } x>1\end{cases}
$$

so that, looking at the signs in the equations of (1.1), we see that $\rho_{1}$ tends to get spread while $\rho_{2}$ is gathering around the origin. But these behaviors are no more preserved for any time, since we have to consider the effect of the coupling. A partially explicit solution can be written as

$$
\begin{aligned}
& \rho_{1}(t, x)= \begin{cases}0 & \text { for } x<0, \\
\frac{\sqrt{2}}{G(t)} & \text { for } 0<x<\frac{1}{\sqrt{2} G(t)}, \\
f(t, x) & \text { for } \frac{1}{\sqrt{2} G(t)}<x<1+t, \\
0 & \text { for } x>1+t,\end{cases} \\
& \rho_{2}(t, x)= \begin{cases}\sqrt{2} G(t) & \text { for } 0<x<\frac{1}{\sqrt{2} G(t)}, \\
0 & \text { otherwise, }\end{cases}
\end{aligned}
$$

where $G(t)=\tanh (\sqrt{2}(t+C))$ and $C=\frac{\sqrt{2}}{2} \tanh ^{-1}\left(\frac{\sqrt{2}}{2}\right)$. Accordingly, we have

$$
h_{\rho}^{\prime}= \begin{cases}\left(\sqrt{2} G(t)-\frac{\sqrt{2}}{G(t)}\right) x & \text { for } 0 \leq x \leq \frac{1}{\sqrt{2} G(t)}, \\ 1-\frac{1}{G^{2}(t)}-\int_{1 /(\sqrt{2} G(t))}^{x} f(t, y) d y & \text { for } \frac{1}{\sqrt{2} G(t)} \leq x \leq 1+t, \\ 0 & \text { otherwise. }\end{cases}
$$

Hence the solution is explicit for $x<1 /(\sqrt{2} G(t))$, before the shock line for $\rho_{2}$ : here $\rho_{1}$ and $\rho_{2}$ satisfy (1.1) because there holds $\dot{G}=\sqrt{2}\left(1-G^{2}\right)$. On the other hand, $f(t, x)$ will be solution to the equation appearing in (3.2) in the region $1 /(\sqrt{2} G(t))<x<1+t$, since in this region there is $\rho_{2}=0$. The corresponding initial values are $\sqrt{2} / G(t)$, given on the curve $x(t)=1 /(\sqrt{2} G(t))$. In this region $\rho_{1}(t, x)=f(t, x)$ decays with time. In particular there will be a linear decay along the characteristics curves $x(t)$, defined by $\frac{d}{d t} x(t)=-h_{\rho_{1}}^{\prime}(t, x(t))$, 
since along such curves there holds

$$
\frac{d}{d t} \rho_{1}(t, x(t))=-\rho_{1}^{2}(t, x(t)) .
$$

We also notice that the conservation of mass implies, for any $t$,

$$
\int_{1 /(\sqrt{2} G(t))}^{1+t} f(t, y) d y=2-\frac{1}{G^{2}(t)} .
$$

Still concerning $\rho_{1}$, we see that in correspondence of $x=1+t$ there holds

$$
\frac{\left[-h_{\rho}^{\prime} \rho_{1}\right]}{\left[\rho_{1}\right]}=1,
$$

so that the shock line has slope equal to 1 and the Rankine-Hugoniot condition is satisfied. Therefore, we have the following picture. $\rho_{1}$ and $\rho_{2}$ tend to the value $\sqrt{2}$ for $x \in[0,1 /(\sqrt{2} G(t))]$, since $\lim _{t \rightarrow+\infty} G(t)=1$ : the common part of mass tends to the equilibrium value $\sqrt{2}$, whereas the exceeding part of mass in $\rho_{1}$ spreads over $\mathbb{R}^{+}$as expected.

Considering the situation $\rho_{0}^{1}(x)=\chi_{[-1,0]}(x), \rho_{0}^{2}(x)=\chi_{[0,1]}(x)$, with

$$
h_{\rho}^{\prime}(t, x)=-\int_{-1}^{x}\left(\rho_{1}(t, y)-\rho_{2}(t, y)\right) d y,
$$

the solution for the model (2.1)-(1.2), as noticed in [8], is $\rho_{1}-\rho_{2}$, where

$$
\rho_{1}(t, x)=\frac{1}{1+t} \chi_{[-1,0]}(x), \quad \rho_{2}(t, x)=\frac{1}{1+t} \chi_{[0,1]}(x) .
$$

In this case $\rho_{1}$ and $\rho_{2}$ are the positive and negative parts of a signed measure, and this behavior is not surprising since it is expected for the two parts to interact and cancel themselves. This can be seen looking at the formulation (2.5), introduced in [2] (see Theorem 6.6 therein). Indeed, from (2.5) it is seen that the total mass of $\rho_{1}$ and $\rho_{2}$ might decrease for effect of $\sigma_{\rho}$. Concerning problem (1.1)-(1.2), we have to expect a different behavior, since the two masses are conserved. But we have to expect the same asymptotic situation, since we have the equilibrium points with $\rho_{1}=\rho_{2}$, hence $\rho=\rho_{1}-\rho_{2}=0$. We search for a solution of the form

$$
\rho^{1}(t, x)=g(t, x) \chi_{[-1, s(t)]}(x), \quad \rho^{2}(t, x)=g(t,-x) \chi_{[-s(t), 1]}(x),
$$

with $-1 \leq s(t) \leq 1$, since the two masses has to keep the symmetry with respect to $x=0$, and the direction of the velocity vector fields keeps the dynamic confined in the interval $(-1,1)$. In particular it is given by

$$
-h_{\rho}^{\prime}= \begin{cases}\int_{-1}^{x} g(t, y) d y & \text { for }-1 \leq x \leq-s(t), \\ \int_{-1}^{-s(t)} g(t, y) d y+\int_{-s(t)}^{x}(g(t, y)-g(t,-y)) d y & \text { for }-s(t) \leq x \leq s(t), \\ \int_{-1}^{-s(t)} g(t, y) d y-\int_{s(t)}^{x} g(t,-y) d y & \text { for } s(t) \leq x \leq 1 .\end{cases}
$$


The solution may be easily explicitly written in the intervals $(-1,-s(t))$ and $(s(t), 1)$, since in these intervals the two parts are not influencing each other yet. Hence

$$
g(t, x)=\frac{1}{1+t} \quad \text { for } x \in(-1,-s(t)) \cup(s(t), 1) .
$$

The symmetry gives

$$
\int_{-1}^{-s(t)} g(t, y) d y+\int_{-s(t)}^{s(t)}(g(t, y)-g(t,-y)) d y=\int_{-1}^{-s(t)} g(t, y) d y=\frac{1-s(t)}{1+t}
$$

and we deduce

$$
-\left.h_{\rho}^{\prime}\right|_{x=s(t)}=\frac{1-s(t)}{1+t} .
$$

$s(t)$ is the shock line, with $s(0)=0$, and the Rankine-Hugoniot condition is then

$$
s^{\prime}(t)=\frac{\left[-h_{\rho}^{\prime} \rho^{1}\right]}{\left[\rho^{1}\right]}=\frac{1-s(t)}{1+t},
$$

entailing $s(t)=t /(1+t)$. Inserting this information in $(3.5)$, it is seen that both $\rho^{1}$ and $\rho^{2}$ tend to occupy the whole interval $(-1,1)$ for large time, and there is indeed a superposition between them. Asymptotically, one expects an equilibrium solution such that $\rho_{1}=\rho_{2}$ (null velocity field), so that $g(t, \cdot)$ tends to an even function.

\section{Existence via Wasserstein variational approach}

In this section we are going to construct a solution to (1.1) as a steepest descent curve of a suitable energy functional, with respect to the 2-Wasserstein metric. This is the approach introduced by F. Otto in the nineties, for the study of the heat equation and the porous medium equation as a gradient flow (see $[14,21])$. Later, it has been exploited for the study of many other models, including superconductivity (see [3]).

We recall some definitions about the Wasserstein structure (see for instance $[1,25,26])$. For $\mu, \nu \in \mathcal{M}_{\alpha}^{2}(\bar{\Omega})$, let $\Gamma(\mu, \nu)$ denote the set of transport plans between them, i.e. measures in $\mathcal{M}_{\alpha}(\bar{\Omega} \times \bar{\Omega})$ whose first and second marginals are respectively $\mu$ and $\nu$. The Wasserstein distance is defined by

$$
W_{2}(\mu, \nu):=\left(\inf _{\gamma \in \Gamma(\mu, \nu)} \int_{\bar{\Omega} \times \bar{\Omega}}|x-y|^{2} d \gamma(x, y)\right)^{1 / 2} .
$$

Here the infimum can be shown to be a minimum, and we let $\Gamma_{0}(\mu, \nu)$ be the class of optimal plans, where this minimum is attained. A transport plan is a generalization of a transport map from $\mu$ to $\nu$, that is, a Borel map $\mathbf{t}$ such that $\mathbf{t}_{\#} \mu=\nu$ (i.e. $\mu\left(\mathbf{t}^{-1}(A)\right)=\nu(A)$, for $A$ Borel). We also recall the classical Brenier result about optimal transport plans (see [4]): if $\gamma \in \Gamma(\mu, \nu)$ and $\mu \ll \mathcal{L}^{2}$, then there exists a unique optimal transport map $\mathbf{t}: \mathbb{R}^{2} \rightarrow \mathbb{R}^{2}$ such that $\gamma=(\mathbf{I}, \mathbf{t})_{\#} \mu$. Moreover, in this case $\mathbf{t}$ is the gradient of a convex function on $\mathbb{R}^{2}$. Finally we recall that, given $\nu \in \mathcal{M}_{\alpha}^{2}(\bar{\Omega})$, the map $\mu \mapsto W_{2}(\nu, \mu)$ is 
l.s.c. in the narrow (or weak) topology of measures, defined by duality with continuous and bounded functions.

Let us now introduce the reference functional. Let $\mu$ belong to $\mathcal{M}(\bar{\Omega})$, the set of real, finite measures over $\bar{\Omega}$. We define

$$
\Phi(\mu):=\frac{1}{2} \int_{\Omega} h_{\mu} d \mu .
$$

Let us discuss some properties of this functional. We first consider the case of a bounded domain $\Omega$. If $\chi_{\Omega} \mu \in H^{-1}(\Omega), h_{\mu}$ is defined through problem (1.2) (indeed it depends only on $\chi_{\Omega} \mu$, so $h_{\mu}=h_{\chi_{\Omega} \mu}$ ). Notice that $\Phi$ itself depends only on the interior part of the measure. From now on, we will make use of the following notation: $\chi_{\Omega} \mu=: \widehat{\mu}$ and $\chi_{\partial \Omega} \mu=: \widetilde{\mu}$ for $\mu \in \mathcal{M}(\bar{\Omega})$. So we have $\Phi(\mu)=\Phi(\widehat{\mu})$. We may think to $\Phi$ as extended with value $+\infty$ if $\chi_{\Omega} \mu \notin H^{-1}(\Omega)$. Now let $\chi_{\Omega} \mu \in H^{-1}(\Omega)$ : an integration by parts using the elliptic equation $-\Delta h_{\mu}=\mu$ and the zero boundary condition yields the alternative formula

$$
\Phi(\mu)=\frac{1}{2} \int_{\Omega}\left|\nabla h_{\mu}\right|^{2} d x
$$

Since $h_{\mu}$ depends linearly on $\mu$, from (4.3) it is clear that $\Phi$ is strictly convex with respect to $\widehat{\mu}$ and nonnegative. If $\nu \in \mathcal{M}(\bar{\Omega})$ is another measure with interior part in $H^{-1}(\Omega)$, the $H_{0}^{1}(\Omega)$ regularity of $h_{\mu}$ and $h_{\nu}$ also yields

$$
\int_{\Omega} h_{\mu} d \nu=\int_{\Omega} h_{\mu} d\left(-\Delta h_{\nu}\right)=\int_{\Omega}\left\langle\nabla h_{\mu}, \nabla h_{\nu}\right\rangle d x=\int_{\Omega} h_{\nu} d\left(-\Delta h_{\mu}\right)=\int_{\Omega} h_{\nu} d \mu .
$$

Let us consider the case $\Omega=\bar{\Omega}=\mathbb{R}^{2}$. In this situation we let $h_{\mu}$ be defined through the fundamental solution of the Laplace equation in two dimensions:

$$
h_{\mu}=-\frac{1}{2 \pi} \int_{\mathbb{R}^{2}} \log |x-y| d \mu(y),
$$

therefore the reference functional (4.2) can be written as

$$
\Phi(\mu)=-\frac{1}{4 \pi} \int_{\mathbb{R}^{2} \times \mathbb{R}^{2}} \log |x-y| d \mu(x) d \mu(y) .
$$

Let now $\mu \in \mathcal{M}\left(\mathbb{R}^{2}\right)$ be such that $\Phi(\mu)<+\infty$. Notice that $h_{\mu}$ does not decay to 0 for $|x| \rightarrow \infty$, unless $\mu\left(\mathbb{R}^{2}\right)=0$. If $\kappa=\mu\left(\mathbb{R}^{2}\right) \neq 0$, let $\mu_{0}$ denote an auxiliary measure, with smooth and compactly supported density and such that $\mu_{0}\left(\mathbb{R}^{2}\right)=\kappa$, and let $h_{0}=-\Delta^{-1} \mu_{0}$. It is known that $h_{0}$ is smooth and enjoys logarithmic behavior at infinity, and now the difference $\mu-\mu_{0}$ belongs to $\mathcal{M}_{0}\left(\mathbb{R}^{2}\right)$, so that $h_{\mu}-h_{0}$ decays at infinity and one may integrate by parts and write

$$
\int_{\mathbb{R}^{2}}\left(h_{\mu}-h_{0}\right) d\left(\mu-\mu_{0}\right)=\int_{\mathbb{R}^{2}}\left(h_{\mu}-h_{0}\right) d\left(-\Delta\left(h_{\mu}-h_{0}\right)\right)=\int_{\mathbb{R}^{2}}\left|\nabla\left(h_{\mu}-h_{0}\right)\right|^{2} d x .
$$


Hence, we obtain

$$
\begin{aligned}
2 \Phi(\mu)=\int_{\mathbb{R}^{2}} h_{\mu} d \mu & =\int_{\mathbb{R}^{2}}\left(h_{\mu}-h_{0}\right) d\left(\mu-\mu_{0}\right)+\int_{\mathbb{R}^{2}}\left(h_{\mu}-h_{0}\right) d \mu_{0}+\int_{\mathbb{R}^{2}} h_{0} d \mu \\
& =\int_{\mathbb{R}^{2}}\left|\nabla\left(h_{\mu}-h_{0}\right)\right|^{2} d x+\int_{\mathbb{R}^{2}}\left(h_{\mu}-h_{0}\right) d \mu_{0}+\int_{\mathbb{R}^{2}} h_{0} d \mu .
\end{aligned}
$$

Using this representation it is not difficult to see that $\Phi$ is again strictly convex. Homogeneity of degree 2 and convexity also yield nonnegativity of $\Phi$; moreover, such properties yield finiteness of $\int_{\mathbb{R}^{2}} h_{\mu} d \nu$ and $\int_{\mathbb{R}^{2}} h_{\nu} d \mu$, as soon as $\Phi(\nu)$ is finite as well. These two quantities are equal, as readily seen from (4.5), and this is true even for a bounded domain $\Omega$, as shown in (4.4). Therefore, in both cases $\Omega$ bounded and $\Omega=\mathbb{R}^{2}$ we may deduce the useful formula for variations of the functional

$$
\Phi(\mu)-\Phi(\nu)=\frac{1}{2} \int_{\Omega} h_{\mu} d \mu-\frac{1}{2} \int_{\Omega} h_{\nu} d \nu=\frac{1}{2} \int_{\Omega}\left(h_{\mu}+h_{\nu}\right) d(\mu-\nu) .
$$

Let us also introduce a particular class of functions, that we will need to prove regularity results. Following [3], we say that $\varphi:[0,+\infty) \rightarrow \mathbb{R}$ is an entropy function if it is nondecreasing, $C^{2}$ and $x \varphi^{\prime}(x)=\varphi(x)$ in $[0,1]$. Moreover, we ask $\varphi$ to enjoy the McCann [19] displacement convexity inequality

$$
2 x^{2} \varphi^{\prime \prime}(x) \geq x \varphi^{\prime}(x)-\varphi(x) .
$$

An example of a $p$-growing entropy is

$$
\varphi(x):= \begin{cases}x & \text { for } 0 \leq x \leq 1, \\ x^{p}+(p-1)\left(1+(p-1) x-\frac{1}{2} p\left(1+x^{2}\right)\right) & \text { for } x>1 .\end{cases}
$$

A possible way to describe steepest descent curves in metric spaces is the minimizing movements scheme. Given $\mu_{1}^{0} \in \mathcal{M}_{\alpha}^{2}(\bar{\Omega}), \mu_{2}^{0} \in \mathcal{M}_{\beta}^{2}(\bar{\Omega})$ such that $\Phi\left(\mu_{1}^{0}-\mu_{2}^{0}\right)<+\infty$ and a time step $\tau>0$, find recursively $\left(\mu_{1}\right)_{\tau}^{k},\left(\mu_{2}\right)_{\tau}^{k}$ among solutions of

$$
\min _{\nu_{1} \in \mathcal{M}_{\alpha}^{2}(\bar{\Omega}), \nu_{2} \in \mathcal{M}_{\beta}^{2}(\bar{\Omega})} \Phi\left(\nu_{1}-\nu_{2}\right)+\frac{1}{2 \tau}\left[W_{2}^{2}\left(\nu_{1},\left(\mu_{1}\right)_{\tau}^{k-1}\right)+W_{2}^{2}\left(\nu_{2},\left(\mu_{2}\right)_{\tau}^{k-1}\right)\right] .
$$

Here we are considering the 2-Wasserstein distance in the product space, while the term $\Phi$, here depending only on the difference $\nu_{1}-\nu_{2}$, accounts for the coupling in (1.1). Very similar minimization problems have been widely studied in $[2,3]$. We can adapt the properties obtained therein. We synthesize them in the following theorem. We skip some details in the proof, since we can mostly refer to the results of these papers. In particular, we address to [2, Sect. 6] for an analysis in the whole plane. Notice that in this last case it is important to work in spaces of measures with finite second moment. This guarantees the right narrow compactness for the existence of minimizers for problem (4.9) and ensures that the suitable integrals over $\mathbb{R}^{2}$ are finite.

Theorem 4.1. Let $\alpha, \beta \geq 0$. Let $\left(\mu_{1}, \mu_{2}\right) \in \mathcal{M}_{\alpha}^{2}(\Omega) \times \mathcal{M}_{\beta}^{2}(\Omega)$ be a starting point for a single step of the minimization problem (4.9), such that $\mu_{1} \in L^{p}(\Omega)$, 
$\mu_{2} \in L^{p}(\Omega), p \geq 4$ (hence $\mu_{1}$ and $\mu_{2}$ have no boundary part). Let $\mu:=\mu_{1}-\mu_{2}$. Then $\Phi(\mu)<+\infty$ and there exists a minimizer $\left(\left(\mu_{1}\right)_{\tau},\left(\mu_{2}\right)_{\tau}\right)$ such that

$$
\left\|\left(\widehat{\mu}_{1}\right)_{\tau}\right\|_{p}+\left\|\left(\widehat{\mu}_{2}\right)_{\tau}\right\|_{p} \leq K_{p},
$$

where $K_{p}$ depends only on $\mu_{1}$ and $\mu_{2}$. Moreover, letting $\mu_{\tau}:=\left(\mu_{1}\right)_{\tau}-\left(\mu_{2}\right)_{\tau}$, such minimizer satisfies

$$
\begin{aligned}
& -\nabla h_{\mu_{\tau}}\left(\widehat{\mu}_{1}\right)_{\tau}=\frac{1}{\tau} \pi_{\#}^{1}\left(\chi_{\Omega}(x)(x-y)\left(\gamma^{1}\right)_{\tau}(x, y)\right) \quad \text { in } \mathcal{D}^{\prime}\left(\mathbb{R}^{2}\right), \\
& \nabla h_{\mu_{\tau}}\left(\widehat{\mu}_{2}\right)_{\tau}=\frac{1}{\tau} \pi_{\#}^{1}\left(\chi_{\Omega}(x)(x-y)\left(\gamma^{2}\right)_{\tau}(x, y)\right) \quad \text { in } \mathcal{D}^{\prime}\left(\mathbb{R}^{2}\right) \text {, }
\end{aligned}
$$

for suitable $\left(\gamma^{1}\right)_{\tau} \in \Gamma_{0}\left(\left(\mu_{1}\right)_{\tau}, \mu_{1}\right)$ and $\left(\gamma^{2}\right)_{\tau} \in \Gamma_{0}\left(\left(\mu_{2}\right)_{\tau}, \mu_{2}\right)$.

Proof. Finiteness of the functional is clear for the case of a bounded domain, since $h_{\mu} \in H_{0}^{1}(\Omega)$. For $\Omega=\mathbb{R}^{2}$ we have by Hölder inequality, since $\mu \in L^{q}$ for any $1 \leq q \leq p$ (here and in the sequel, $\mu$ will denote both the measure and its density),

$$
\begin{aligned}
4 \pi \Phi(\mu) & =-\int_{|x-y|<1} \log |x-y| d \mu(x) d \mu(y)-\int_{|x-y| \geq 1} \log |x-y| d \mu(x) d \mu(y) \\
& \leq\|\mu\|_{2}^{2} \int_{-1}^{1} \log |r| d r+\int_{|x-y| \geq 1}|x-y||\mu(x)||\mu(y)| d x d y .
\end{aligned}
$$

Notice that the last term is bounded thanks to the finiteness of the second moments of $\mu_{1}$ and $\mu_{2}$. We may also deduce boundedness of $\nabla h_{\mu}$. Indeed, denoting by $B_{1}$ the unit ball in $\mathbb{R}^{2}$ centered in the origin, we have

$$
\begin{aligned}
4 \pi\left|\nabla h_{\mu}(x)\right| & =\left|\int_{\mathbb{R}^{2}} \frac{x-y}{|x-y|^{2}} d \mu(y)\right| \leq \int_{B_{1}} \frac{|\mu(x-y)|}{|y|} d y+\int_{\mathbb{R}^{2} \backslash B_{1}} \frac{|\mu(x-y)|}{|y|} d y \\
& \leq\left(\int_{B_{1}} \frac{d y}{|y|^{4 / 3}}\right)^{\frac{3}{4}}\left(\int_{B_{1}}|\mu(x-y)|^{4} d y\right)^{\frac{1}{4}}+\int_{\mathbb{R}^{2} \backslash B_{1}}|\mu(x-y)| d y \\
& \leq(3 \pi)^{3 / 4}\|\mu\|_{4}+\alpha+\beta .
\end{aligned}
$$

Let us analyze the one step minimization: it is useful to introduce a regularized problem, in order to get $L^{p}$ regularity of minimizers:

$$
\begin{aligned}
\min _{\nu_{1} \in \mathcal{M}_{\alpha}^{2}(\bar{\Omega}), \nu_{2} \in \mathcal{M}_{\beta}^{2}(\bar{\Omega})} \Phi\left(\nu_{1}-\nu_{2}\right)+ & \frac{1}{2 \tau}\left[W_{2}^{2}\left(\nu_{1}, \mu_{1}\right)+W_{2}^{2}\left(\nu_{2}, \mu_{2}\right)\right] \\
& +\delta \int_{\Omega}\left|\nu_{1}\right|^{4}+\delta \int_{\Omega}\left|\nu_{2}\right|^{4} .
\end{aligned}
$$

Existence of a solution $\left(\left(\mu_{1}\right)_{\tau}^{\delta},\left(\mu_{2}\right)_{\tau}^{\delta}\right) \in \mathcal{M}_{\alpha}^{2}(\bar{\Omega}) \times \mathcal{M}_{\beta}^{2}(\bar{\Omega})$ is ensured by tightness and lower semicontinuity, and the penalization term gives the $L^{4}(\Omega)$ regularity of its interior part. It is not difficult to show, using the semicontinuity of $W_{2}(\cdot, \mu)$, that, as $\delta \rightarrow 0$, there exists a limit point $\left(\left(\mu_{1}\right)_{\tau},\left(\mu_{2}\right)_{\tau}\right)$ of $\left(\left(\mu_{1}\right)_{\tau}^{\delta},\left(\mu_{2}\right)_{\tau}^{\delta}\right)$, in the narrow topology of measures, which minimizes (4.9).

Let us consider a variation starting from the minimizer $\left(\left(\mu_{1}\right)_{\tau}^{\delta},\left(\mu_{2}\right)_{\tau}^{\delta}\right)$ of (4.13). For simplicity of notation, we denote it by $\left(\nu_{1}, \nu_{2}\right)$. We let $\boldsymbol{\xi} \in$ $C_{c}^{\infty}\left(\Omega ; \mathbb{R}^{2}\right)$ and $\nu_{1, \varepsilon}=(\mathbf{I}+\varepsilon \boldsymbol{\xi})_{\#} \widehat{\nu}_{1}+\widetilde{\nu}$. Hence we are perturbing the interior 


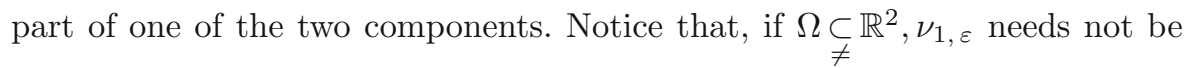
supported in $\bar{\Omega}$. Nevertheless, one can always minimize among measures over the whole plane and than construct a minimizer supported in $\bar{\Omega}$ simply projecting on the boundary along the shortest line segment: such minimizer will satisfy the same Euler Lagrange equations (4.11), since only its interior part plays a role therein (for the details on this argument, we refer to [2, Corollary $3.3])$. In particular we are always thinking to $\widehat{\nu}_{1}, \widehat{\nu}_{2}$ as extended with value 0 outside $\Omega$.

Since $\widehat{\nu}_{1} \in L^{4}(\Omega), \widehat{\nu}_{1, \varepsilon}$ also belongs to $L^{4}(\Omega)$ and there holds

$$
\widehat{\nu}_{1, \varepsilon}=\frac{\widehat{\nu}_{1}}{\operatorname{det}(I+\varepsilon D \boldsymbol{\xi})} \circ\left(\left.[(\mathbf{I}+\varepsilon \boldsymbol{\xi})]^{-1}\right|_{(\mathbf{I}+\varepsilon \boldsymbol{\xi})\left(\Omega_{\varepsilon}\right)}\right),
$$

where $\Omega_{\varepsilon}:=\{x \in \Omega: x+\varepsilon \boldsymbol{\xi}(x) \in \Omega\}$. Hence,

$$
\int_{\Omega}\left(\widehat{\nu}_{1, \varepsilon}-\widehat{\nu}_{1}\right)^{4}=\int_{\Omega}\left(\frac{\widehat{\nu}_{1}}{\operatorname{det}(I+\varepsilon D \boldsymbol{\xi})} \circ\left(\left.[(\mathbf{I}+\varepsilon \boldsymbol{\xi})]^{-1}\right|_{(\mathbf{I}+\varepsilon \boldsymbol{\xi})\left(\Omega_{\varepsilon}\right)}\right)-\widehat{\nu}_{1}\right)^{4},
$$

so that $\widehat{\nu}_{1, \varepsilon} \rightarrow \widehat{\nu}_{1}$ in $L^{4}(\Omega)$. This is one of the key points: as a consequence we have (by elliptic regularity, one may refer to the standard texts like [12] or [13]) the strong $W^{2,4}(\Omega)$ convergence of $h_{\nu_{1, \varepsilon}-\nu_{2}}$ to $h_{\nu_{1}-\nu_{2}}$ (on compact sets for the case $\Omega=\mathbb{R}^{2}$ ) and, by Sobolev embedding, the strong $C^{1}(\bar{\Omega})$ convergence.

Now let $\nu:=\nu_{1}-\nu_{2}$ and $\nu_{\varepsilon}:=\nu_{1, \varepsilon}-\nu_{2}$. Making use of (4.6) we see that $2 \Phi\left(\nu_{\varepsilon}\right)-2 \Phi(\nu)$ may be written as

$$
\begin{aligned}
\int_{\Omega}\left(h_{\nu_{\varepsilon}}+h_{\nu}\right) d\left(\widehat{\nu}_{\varepsilon}-\widehat{\nu}\right)= & \int_{\Omega_{\varepsilon}}\left(h_{\nu_{\varepsilon}} \circ(\mathbf{I}+\varepsilon \boldsymbol{\xi})+h_{\nu} \circ(\mathbf{I}+\varepsilon \boldsymbol{\xi})\right) d \widehat{\nu}_{1}-\int_{\Omega}\left(h_{\nu_{\varepsilon}}+h_{\nu}\right) d \widehat{\nu}_{1} \\
= & \int_{\Omega}\left(h_{\nu_{\varepsilon}} \circ(\mathbf{I}+\varepsilon \boldsymbol{\xi})-h_{\nu_{\varepsilon}}+h_{\nu} \circ(\mathbf{I}+\varepsilon \boldsymbol{\xi})-h_{\nu}\right) d \widehat{\nu}_{1} \\
& -\int_{\Omega \backslash \Omega_{\varepsilon}}\left(h_{\nu_{\varepsilon}} \circ(\mathbf{I}+\varepsilon \boldsymbol{\xi})+h_{\nu} \circ(\mathbf{I}+\varepsilon \boldsymbol{\xi})\right) d \widehat{\nu}_{1} .
\end{aligned}
$$

But the $C^{1}(\bar{\Omega})$ regularity of $h_{\nu}$ and the fact that $h_{\nu}=0$ on $\partial \Omega$ imply that the last term is $o(\varepsilon)$ as $\varepsilon \rightarrow 0$. We find (even for $\Omega=\mathbb{R}^{2}$, since $\boldsymbol{\xi}$ is compactly supported)

$$
\Phi\left(\nu_{\varepsilon}\right)-\Phi(\nu)=\varepsilon \int_{\Omega}\left\langle\nabla h_{\nu}, \boldsymbol{\xi}\right\rangle d \nu_{1}+o(\varepsilon) .
$$

For the regularizing term, we make use of the change of variables,

$$
\begin{aligned}
\delta\left[\int_{\Omega}\left|\widehat{\nu}_{1, \varepsilon}\right|^{4}-\int_{\Omega}|\widehat{\nu}|^{4}\right] & =\delta\left[\int_{\Omega_{\varepsilon}} \frac{\widehat{\nu}_{1}^{4}}{\operatorname{det}^{3}(I+\varepsilon D \boldsymbol{\xi})}-\int_{\Omega} \widehat{\nu}_{1}^{4}\right] \\
& \leq-3 \delta \varepsilon \int_{\Omega} \widehat{\nu}_{1}^{4} \nabla \cdot \boldsymbol{\xi}+o(\varepsilon) .
\end{aligned}
$$

Next consider a plan $\gamma^{1} \in \Gamma_{0}\left(\nu_{1}, \mu_{1}\right)$ and the plan $(\mathbf{I}+\varepsilon \boldsymbol{\xi}, \mathbf{I})_{\#}\left(\chi_{\Omega \times \bar{\Omega}} \gamma^{1}\right)+$ $\chi_{\partial \Omega \times \bar{\Omega}} \gamma^{1} \in \Gamma\left(\nu_{1, \varepsilon}, \mu_{1}\right)$. Clearly we have

$$
W_{2}^{2}\left(\nu_{1, \varepsilon}, \mu_{1}\right)-W_{2}^{2}\left(\nu_{1}, \mu_{1}\right) \leq 2 \varepsilon \int_{\Omega \times \bar{\Omega}} \boldsymbol{\xi}(x)(x-y) d \gamma^{1}(x, y)+o(\varepsilon) .
$$


Combining (4.14), (4.15) and (4.16), dividing by $\varepsilon$, thanks to the minimality of $\left(\nu_{1}, \nu_{2}\right)$ we get

$$
-3 \delta \int_{\Omega} \widehat{\nu}_{1}^{4} \nabla \cdot \boldsymbol{\xi}+\int_{\Omega} \nabla h_{\nu} \cdot \boldsymbol{\xi} d \nu_{1}+\frac{1}{\tau} \int_{\bar{\Omega}} \boldsymbol{\xi} \cdot d\left[\pi_{\#}^{1}\left(\chi_{\Omega}(x)(x-y) \gamma^{1}\right)\right] \geq 0,
$$

Changing $\boldsymbol{\xi}$ in $-\boldsymbol{\xi}$ we get equality, that is

$$
-3 \delta \nabla\left(\widehat{\nu}_{1}^{4}\right)-\nabla h_{\nu} \widehat{\nu}_{1}=\frac{1}{\tau} \pi_{\#}^{1}\left(\chi_{\Omega \times \bar{\Omega}}(x-y) \gamma^{1}\right) .
$$

One makes the same kind of variation on $\nu_{2}$, with $\nu_{1}$ fixed, and obtains

$$
-3 \delta \nabla\left(\widehat{\nu}_{2}^{4}\right)+\nabla h_{\nu} \widehat{\nu}_{2}=\frac{1}{\tau} \pi_{\#}^{1}\left(\chi_{\Omega \times \bar{\Omega}}(x-y) \gamma^{2}\right),
$$

where $\gamma^{2} \in \Gamma_{0}\left(\nu_{2}, \mu_{2}\right)$. We see that these are the perturbed versions of the equations (4.11).

We need to pass to the limit and obtain (4.11). Let us reason on (4.17). Since $\widehat{\nu}_{1} \ll \mathcal{L}^{2}$, we know by Brenier's theorem [4] that $\chi_{\Omega \times \bar{\Omega}} \gamma^{1}$ is a plan induced by an optimal transport map $\mathbf{r}_{1}$. Similarly a map $\mathbf{r}_{2}$ corresponds to $\chi_{\Omega \times \bar{\Omega}} \gamma^{2}$, and these maps are the gradients of two convex Lipschitz functions (defined on $\mathbb{R}^{2}$ ). We may also assume that $\mathbf{r}_{1}, \mathbf{r}_{2}$ are bounded: this is always true if $\mu_{1}, \mu_{2}$ are compactly supported, otherwise we would need an approximation argument for which we refer to [2, Lemma 6.4]. Therefore we have $\mathbf{r}_{1}, \mathbf{r}_{2} \in B V_{l o c}\left(\mathbb{R}^{2}\right) \cap L^{\infty}\left(\mathbb{R}^{2}\right)$ and

$$
\pi_{\#}^{1}\left(\chi_{\Omega}(x)(x-y) \gamma^{1}\right)=\left(\mathbf{I}-\mathbf{r}_{1}\right) \widehat{\nu}_{1}, \quad \pi_{\#}^{1}\left(\chi_{\Omega}(x)(x-y) \gamma^{2}\right)=\left(\mathbf{I}-\mathbf{r}_{2}\right) \widehat{\nu}_{2} .
$$

This way (4.17) becomes

$$
-3 \delta \nabla\left(\widehat{\nu}_{1}^{4}\right)-\nabla h_{\nu} \widehat{\nu}_{1}=\frac{1}{\tau}\left(\mathbf{I}-\mathbf{r}_{1}\right) \widehat{\nu}_{1} \quad \text { in } \mathcal{D}^{\prime}\left(\mathbb{R}^{2}\right) .
$$

Since $\mathbf{r}_{1}, \mathbf{r}_{2} \in L^{\infty}\left(\mathbb{R}^{2}\right)$, the right hand side is in $L^{4}\left(\mathbb{R}^{2}\right)$. But since $\nabla h_{\nu}$ is continuous and bounded (recalling (4.12) if $\Omega=\mathbb{R}^{2}$ ), we have $\nabla h_{\nu} \widehat{\nu}_{1} \in L^{4}\left(\mathbb{R}^{2}\right)$, so that by comparison in (4.19) we find $\widehat{\nu}_{1}^{4} \in W^{1,4}\left(\mathbb{R}^{2}\right)$, and by Sobolev embedding $\widehat{\nu}_{1}$ is continuous on $\mathbb{R}^{2}$. Mind that $\widehat{\nu}_{1}$ is also vanishing outside $\Omega$. Analogously $\widehat{\nu}_{2}$ is continuous. Let now $\varphi$ be an entropy function. The basic inequality following from displacement convexity is found in [1, Lemma 10.4.4] and in this case it reads

$$
\int_{\mathbb{R}^{2}} \varphi\left(\mathbf{r}_{1 \#} \widehat{\nu}_{1}\right)-\varphi\left(\widehat{\nu}_{1}\right) \geq-\int_{\mathbb{R}^{2}} \psi^{\prime}\left(\widehat{\nu}_{1}\right) \operatorname{tr}\left(\nabla\left(\mathbf{r}_{1}-\mathbf{I}\right)\right),
$$

where $\psi$ is the even convex function on $\mathbb{R}$ defined by $\psi^{\prime}(x):=x \varphi^{\prime}(x)-\varphi(x)$

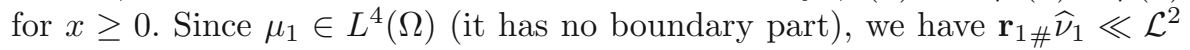
and $\mathbf{r}_{1 \#} \widehat{\nu}_{1} \leq \mu_{1}$, so that by the previous formula we deduce

$$
\int_{\mathbb{R}^{2}} \varphi\left(\mu_{1}\right)-\varphi\left(\widehat{\nu}_{1}\right) \geq-\int_{\mathbb{R}^{2}} \psi^{\prime}\left(\widehat{\nu}_{1}\right) \operatorname{tr}\left(\nabla\left(\mathbf{r}_{1}-\mathbf{I}\right)\right) .
$$

But $\mathbf{r}_{1}$ is the gradient of a convex function, so that we have $\operatorname{tr}\left(\nabla\left(\mathbf{r}_{1}-\mathbf{I}\right)\right) \leq$ $\operatorname{div}\left(\mathbf{r}_{1}-\mathbf{I}\right)$ (the divergence has to be understood in the distributional sense, 
and it is a measures since $\mathbf{r}_{1}$ is $B V$ ). But this can be computed using (4.19), hence

$$
\int_{\mathbb{R}^{2}} \varphi\left(\mu_{1}\right)-\varphi\left(\widehat{\nu}_{1}\right) \geq-\tau \int_{\mathbb{R}^{2}} \psi^{\prime}\left(\widehat{\nu}_{1}\right) \operatorname{div}\left[3 \delta \frac{\nabla\left(\widehat{\nu}_{1}^{4}\right)}{\widehat{\nu}_{1}}+\nabla h_{\nu}\right] .
$$

Starting from (4.18), the same arguments provide the corresponding inequality for $\nu_{2}$, that is

$$
\int_{\mathbb{R}^{2}} \varphi\left(\mu_{2}\right)-\varphi\left(\widehat{\nu}_{2}\right) \geq-\tau \int_{\mathbb{R}^{2}} \psi^{\prime}\left(\widehat{\nu}_{2}\right) \operatorname{div}\left[3 \delta \frac{\nabla\left(\widehat{\nu}_{2}^{4}\right)}{\widehat{\nu}_{2}}-\nabla h_{\nu}\right] .
$$

But $-\Delta h_{\nu}=\nu$, and since $\psi^{\prime}$ is nondecreasing we have

$$
\int_{\mathbb{R}^{2}} \psi^{\prime}\left(\widehat{\nu}_{1}\right) \operatorname{div}\left(\nabla h_{\nu}\right)-\int_{\mathbb{R}^{2}} \psi^{\prime}\left(\widehat{\nu}_{2}\right) \operatorname{div}\left(\nabla h_{\nu}\right)=\int_{\mathbb{R}^{2}}\left(\psi^{\prime}\left(\widehat{\nu}_{1}\right)-\psi^{\prime}\left(\widehat{\nu}_{2}\right)\right)\left(\widehat{\nu}_{2}-\widehat{\nu}_{1}\right) \leq 0 \text {. }
$$

On the other hand, the convexity of $\psi$ and an integration by parts yield

$$
\int_{\mathbb{R}^{2}} \psi^{\prime}\left(\widehat{\nu}_{1}\right) \operatorname{div}\left(\frac{\nabla\left(\widehat{\nu}_{1}^{4}\right)}{\widehat{\nu}_{1}}\right)=-4 \int_{\mathbb{R}^{2}} \nabla \psi^{\prime}\left(\widehat{\nu}_{1}\right) \cdot\left(\widehat{\nu}_{1}^{2} \nabla \widehat{\nu}_{1}\right)=-\int_{\mathbb{R}^{2}} \psi^{\prime \prime}\left(\widehat{\nu}_{1}\right) \widehat{\nu}_{1}^{2}\left|\nabla \widehat{\nu}_{1}\right|^{2} \leq 0 .
$$

Here the control on the boundary term comes from the vanishing of $\widehat{\nu}_{1}$ outside $\Omega$. In the case $\Omega=\mathbb{R}^{2}$, it comes from the $W^{1,4}\left(\mathbb{R}^{2}\right)$ regularity of $\widehat{\nu}_{1}^{4}$, yielding vanishing at infinity of $\widehat{\nu}_{1}$ by standard Sobolev imbedding results: since $\psi^{\prime}$ vanishes in a whole interval containing the origin, $\psi^{\prime}\left(\widehat{\nu}_{1}\right)$ is still compactly supported. Similarly the analogous integral involving $\widehat{\nu}_{2}$ is nonpositive. Summing (4.20) and (4.21) and taking advantage of these last inequalities we get

$$
\int_{\Omega} \varphi\left(\mu_{1}\right)+\varphi\left(\mu_{2}\right)-\varphi\left(\widehat{\nu}_{1}\right)-\varphi\left(\widehat{\nu}_{2}\right) \geq 0 .
$$

If $\varphi$ is chosen to be a $p$-growing entropy like (4.8), this shows that $\widehat{\nu}_{1}=\left(\widehat{\mu}_{1}\right)_{\tau}^{\delta}$ and $\widehat{\nu}_{2}=\left(\widehat{\mu}_{2}\right)_{\tau}^{\delta}$ are uniformly bounded (in $\tau$ and $\delta$ ) in $L^{p}(\Omega)$ (the bounds depending only on $\mu_{1}$ and $\mu_{2}$ ). We know that there exists a vanishing sequence $\delta_{n}$ such that $\left(\mu_{1}\right)_{\tau}^{\delta_{n}}$ and $\left(\mu_{2}\right)_{\tau}^{\delta_{n}}$ converge weakly in measure respectively to $\left(\mu_{1}\right)_{\tau}$ and $\left(\mu_{2}\right)_{\tau}$, a couple which minimizes (4.9). But we just learned that (the interior parts of) these sequences are bounded in $L^{p}(\Omega)$, hence the weak lower semicontinuity of the map $\widehat{\mu} \mapsto \int_{\Omega} \varphi(\widehat{\mu})$ allows to deduce (4.10) for $4 \leq p<+\infty$. The $L^{\infty}$ case is obtained with a simple approximation argument (see [2, Corollary 4.3 and Corollary 6.5]). Since a $L^{p}$ minimizer has been found for (4.9), such minimizer is regular enough to apply the same variational arguments of the first part of this proof to problem (4.9) itself and find the unperturbed Euler-Lagrange equations (4.11).

Consider now an initial datum $\left(\mu_{1}^{0}, \mu_{2}^{0}\right) \in \mathcal{M}_{\alpha}^{2}(\bar{\Omega}) \times \mathcal{M}_{\beta}^{2}(\bar{\Omega})$, such that $\Phi\left(\mu_{1}^{0}-\mu_{2}^{0}\right)<+\infty$. Starting from these measures, we apply the minimizing movements scheme. Since we want to work in the framework of Theorem 4.1, at each step we start from the interior part of the previous minimizer (of course this distinction is unnecessary if $\Omega=\mathbb{R}^{2}$ ). That is, we search recursively for 
$\left(\left(\nu_{1}\right)_{\tau}^{k},\left(\nu_{2}\right)_{\tau}^{k}\right)$ among solutions of $\min _{\nu_{1} \in \mathcal{M}_{\alpha_{k}}^{2}(\bar{\Omega}), \nu_{2} \in \mathcal{M}_{\beta_{k}}^{2}(\bar{\Omega})} \Phi\left(\nu_{1}-\nu_{2}\right)+\frac{1}{2 \tau}\left[W_{2}^{2}\left(\nu_{1},\left(\widehat{\mu}_{1}\right)_{\tau}^{k-1}\right)+W_{2}^{2}\left(\nu_{2},\left(\widehat{\mu}_{2}\right)_{\tau}^{k-1}\right)\right]$,

where $\alpha_{k}=\left(\widehat{\mu}_{1}\right)_{\tau}^{k-1}(\Omega) \leq \alpha$ and $\beta_{k}=\left(\widehat{\mu}_{2}\right)_{\tau}^{k-1}(\Omega) \leq \beta$. In particular, for any $k$ we consider a solution satisfying the properties of Theorem 4.1 (whose existence is ensured by the same theorem). Notice also that the step by step entropy control therein, which is (4.22), ensures that the bound (4.10) holds for a constant $K_{p}$ which does not depend on $k$, but only on the initial datum $\left(\mu_{1}^{0}, \mu_{2}^{0}\right)$. We then let

$$
\left(\mu_{1}\right)_{\tau}^{k}=\left(\nu_{1}\right)_{\tau}^{k}+\left(\widetilde{\mu}_{1}\right)_{\tau}^{k-1}, \quad\left(\mu_{2}\right)_{\tau}^{k}=\left(\nu_{2}\right)_{\tau}^{k}+\left(\widetilde{\mu}_{2}\right)_{\tau}^{k-1} .
$$

This way, in the scheme there can be mass transported to the boundary, but the mass on the boundary can not enter in the domain in the subsequent steps (this dynamic is the same of $[2,3]$ ). Notice also that the choice of $\left(\left(\nu_{1}\right)_{\tau}^{k},\left(\nu_{2}\right)_{\tau}^{k}\right)$ implies that even $\left(\left(\mu_{1}\right)_{\tau}^{k},\left(\mu_{2}\right)_{\tau}^{k}\right)$, for any $k$, satisfies $(4.10)$ with the same constant $K_{p}$ independent of $k$. Now we have two sequences

$$
\left(\left(\mu_{1}\right)_{\tau}^{k}\right) \subset \mathcal{M}_{\alpha}^{2}(\bar{\Omega}), \quad\left(\left(\mu_{2}\right)_{\tau}^{k}\right) \subset \mathcal{M}_{\beta}^{2}(\bar{\Omega}) .
$$

We construct piecewise constant interpolations:

$$
\left(\bar{\mu}_{1}\right)_{\tau}(t):=\left(\mu_{1}\right)_{\tau}^{\lceil t / \tau\rceil} \text { and }\left(\bar{\mu}_{2}\right)_{\tau}(t):=\left(\mu_{2}\right)_{\tau}^{\lceil t / \tau\rceil},
$$

where $\lceil\cdot\rceil$ denotes the (superior) integer part. We then pass to the limit as $\tau$ goes to 0 . We have the following

Proposition 4.1. As $\tau \rightarrow 0$, there exists two weakly continuous families of measures $t \mapsto \mu_{1}(t) \in \mathcal{M}_{\alpha}^{2}(\bar{\Omega})$ and $t \mapsto \mu_{2}(t) \in \mathcal{M}_{\beta}^{2}(\bar{\Omega})$ such that, weakly in the sense of measures, on a suitable subsequence,

$$
\left(\bar{\mu}_{1}\right)_{\tau}(t) \rightarrow \mu_{1}(t), \quad\left(\bar{\mu}_{2}\right)_{\tau}(t) \rightarrow \mu_{2}(t), \quad \forall t \geq 0 .
$$

Proof. The minimality of $\left(\left(\nu_{1}\right)_{\tau}^{k},\left(\nu_{2}\right)_{\tau}^{k}\right)$, starting from $\left(\left(\widehat{\mu}_{1}\right)_{\tau}^{k-1},\left(\widehat{\mu}_{2}\right)_{\tau}^{k-1}\right)$, easily entails

$$
\sum_{k=m+1}^{n}\left[W_{2}^{2}\left(\left(\nu_{1}\right)_{\tau}^{k},\left(\widehat{\mu}_{1}\right)_{\tau}^{k-1}\right)+W_{2}^{2}\left(\left(\nu_{2}\right)_{\tau}^{k},\left(\widehat{\mu}_{2}\right)_{\tau}^{k-1}\right)\right] \leq 2 \tau \Phi\left(\mu_{1}^{0}-\mu_{2}^{0}\right), \quad \forall m, n \in \mathbb{N},
$$

and since the 2-Wasserstein distance does not increase adding the same measure to the source and to the target, we get the basic estimate

$$
\sum_{k=m+1}^{n}\left[W_{2}^{2}\left(\left(\mu_{1}\right)_{\tau}^{k},\left(\mu_{1}\right)_{\tau}^{k-1}\right)+W_{2}^{2}\left(\left(\mu_{2}\right)_{\tau}^{k},\left(\mu_{2}\right)_{\tau}^{k-1}\right)\right] \leq 2 \tau \Phi\left(\mu_{1}^{0}-\mu_{2}^{0}\right) .
$$

By the triangle inequality we get

$$
\begin{aligned}
& W_{2}^{2}\left(\left(\mu_{1}\right)_{\tau}^{n+1},\left(\mu_{1}\right)_{\tau}^{m}\right) \leq 2 \tau(n-m) \Phi\left(\mu_{1}^{0}-\mu_{2}^{0}\right), \\
& W_{2}^{2}\left(\left(\mu_{2}\right)_{\tau}^{n+1},\left(\mu_{2}\right)_{\tau}^{m}\right) \leq 2 \tau(n-m) \Phi\left(\mu_{1}^{0}-\mu_{2}^{0}\right) .
\end{aligned}
$$


Consider the first of these inequalities: we may connect the various points $m, n$ with continuous curves (for the details we refer to [1, Theorem 11.1.6]) and obtain a family $t \mapsto\left(\breve{\mu}_{1}\right)_{\tau}(t) \in \mathcal{M}_{\alpha}^{2}(\bar{\Omega})$ such that, for any $t \geq s$,

$$
W_{2}\left(\left(\breve{\mu}_{1}\right)_{\tau}(t),\left(\breve{\mu}_{1}\right)_{\tau}(s)\right) \leq \sqrt{2 \Phi\left(\mu_{1}^{0}-\mu_{2}^{0}\right)(t-s)} .
$$

Hence this family (parametrized by $\tau$ ) of functions is equicontinuous with respect to the narrow topology of measures. An application of the Ascoli-Arzelá theorem yields the pointwise weak convergence (on a suitable subsequence) to some $\mu_{1}(t) \in \mathcal{M}_{\alpha}^{2}(\bar{\Omega})$. In order to conclude, notice that by $(4.25)$ we also have

$$
W_{2}\left(\left(\bar{\mu}_{1}\right)_{\tau}(t),\left(\breve{\mu}_{1}\right)_{\tau}(t)\right) \leq \sqrt{2 \tau \Phi\left(\mu_{1}^{0}-\mu_{2}^{0}\right)}+W_{2}\left(\left(\breve{\mu}_{1}\right)_{\tau}(t), \mu_{1}(t)\right)
$$

so that $\left(\bar{\mu}_{1}\right)_{\tau}(t)$ also converge to $\mu_{1}(t)$. Similarly one shows the convergence of $\left(\bar{\mu}_{2}\right)_{\tau}(t)$ to $\mu_{2}(t)$.

Remark 4.1. If $\widehat{\mu}_{1}^{0}, \widehat{\mu}_{2}^{0}$ belong to $L^{p}(\Omega), p \geq 4$, thanks to the $L^{p}$ estimates obtained in Theorem 4.1 (and recalling that $\left(\left(\mu_{1}\right)_{\tau}^{k},\left(\mu_{2}\right)_{\tau}^{k}\right)$ satisfy (4.10) with the constant $K_{p}$ independent of $k$ ) we infer that $\widehat{\mu}_{1}(t), \widehat{\mu}_{2}(t)$ are uniformly bounded in $L^{p}(\Omega)$.

We let $\mu(t)$ be the weak limit of the difference $\left(\bar{\mu}_{1}\right)_{\tau}(t)-\left(\bar{\mu}_{2}\right)_{\tau}(t)$. Then, for each $t$ we have $\mu_{1}(t)-\mu_{2}(t)=\mu(t)$. In general $\mu_{1}$ and $\mu_{2}$ are not the positive and negative parts of $\mu$, the overlapping being possible also in the weak limit. Now we can quickly deduce the continuity equations satisfied by $\mu_{1}(t), \mu_{2}(t)$, therefore proving the main existence result.

\section{Proof of Theorem 1.1}

Let $\left(\mu_{1}^{0}, \mu_{2}^{0}\right) \in \mathcal{M}_{\alpha}^{2}(\bar{\Omega}) \times \mathcal{M}_{\beta}^{2}(\bar{\Omega})$ and $\widehat{\mu}_{1}^{0}, \widehat{\mu}_{2}^{0} \in L^{p}(\Omega), p \geq 4$. We are going to show that the families of measures $\mu_{1}(t), \mu_{2}(t)$, constructed in Proposition 4.1 and starting from $\mu_{1}^{0}, \mu_{2}^{0}$, are a distributional solution to (1.1)-(1.2). In particular, the regularity estimates above (see Remark 4.1) give

$$
\left\|\widehat{\mu}_{1}(t)\right\|_{p}+\left\|\widehat{\mu}_{2}(t)\right\|_{p} \leq K_{p} \quad \forall t \geq 0,
$$

where $K_{p}$ is a suitable positive constant depending only on the initial data. Moreover, by the discrete construction the parts of mass from $\mu_{1}$ and $\mu_{2}$ which get cumulated on the boundary at each step do not play any role in the subsequent time steps: in the dynamic we are describing, we see that $t \mapsto \mu_{1}(t)(\Omega)$ and $t \mapsto \widehat{\mu}_{2}(t)(\Omega)$ are therefore nonincreasing maps.

In order to work with a simpler notation, we limit ourselves to prove the case $\Omega=\mathbb{R}^{2}$. The case of a domain can be treated in the same way, and with a simple adaptation of the arguments contained in Proposition 5.4 and Theorem 5.6 of [2]. In the same spirit of [14], we have in the sense of distributions, for $\varphi \in C_{0}^{2}\left(\mathbb{R}^{2}\right)$,

$$
\frac{d}{d t} \int_{\mathbb{R}^{2}} \varphi d\left(\bar{\mu}_{1}\right)_{\tau}(t)=\sum_{k=0}^{+\infty}\left(\int_{\mathbb{R}^{2}} \varphi d\left(\mu_{1}\right)_{\tau}^{k+1}-\int_{\mathbb{R}^{2}} \varphi d\left(\mu_{1}\right)_{\tau}^{k}\right) \delta_{\tau k}(t)
$$


Now we change variables and we make use of the first equation in (4.11), written for the discrete minimizer $\left(\left(\mu_{1}\right)_{\tau}^{k+1},\left(\mu_{2}\right)_{\tau}^{k+1}\right)$ starting from the previous minimizer $\left(\left(\mu_{1}\right)_{\tau}^{k},\left(\mu_{2}\right)_{\tau}^{k}\right)$, so that in this case it reads $-\nabla h_{\mu_{\tau}^{k+1}}\left(\mu_{1}\right)_{\tau}^{k+1}=$ $\frac{1}{\tau} \pi_{\#}^{1}\left((x-y)\left(\gamma^{1}\right)_{\tau}^{k+1}\right)$; this way from $(4.26)$ we obtain

$$
\begin{aligned}
\int_{\mathbb{R}^{2}} \varphi d\left(\mu_{1}\right)_{\tau}^{k+1}-\int_{\mathbb{R}^{2}} \varphi d\left(\mu_{1}\right)_{\tau}^{k} & =\int_{\mathbb{R}^{2}} \varphi d \pi_{\#}^{1}\left(\gamma^{1}\right)_{\tau}^{k+1}-\int_{\mathbb{R}^{2}} \varphi d \pi_{\#}^{2}\left(\gamma^{1}\right)_{\tau}^{k+1} \\
& =\int_{\mathbb{R}^{2} \times \mathbb{R}^{2}}(\varphi(x)-\varphi(y)) d\left(\gamma^{1}\right)_{\tau}^{k+1}(x, y) \\
& =\int_{\mathbb{R}^{2} \times \mathbb{R}^{2}}\langle\nabla \varphi(x), x-y\rangle d\left(\gamma^{1}\right)_{\tau}^{k+1}(x, y)+\left(\mathbf{R}_{1}\right)_{\tau}^{k+1} \\
& =-\tau \int_{\mathbb{R}^{2}}\left\langle\nabla \varphi, \nabla h_{\mu_{\tau}^{k+1}}\right\rangle d\left(\mu_{1}\right)_{\tau}^{k+1}+\left(\mathbf{R}_{1}\right)_{\tau}^{k+1}
\end{aligned}
$$

where $\left(\gamma^{1}\right)_{\tau}^{k+1} \in \Gamma_{0}\left(\left(\mu_{1}\right)_{\tau}^{k+1},\left(\mu_{1}\right)_{\tau}^{k}\right)$ and $\left(\mathbf{R}_{1}\right)_{\tau}^{k+1}$ is the remainder of the Taylor expansion:

$$
\left(\mathbf{R}_{1}\right)_{\tau}^{k+1}=-\frac{1}{2} \int_{0}^{1} \int_{\mathbb{R}^{2}}\left\langle\nabla^{2}((1-\vartheta) x+\vartheta y)(y-x), y-x\right\rangle d\left(\gamma^{1}\right)_{\tau}^{k+1}(x, y) d \vartheta
$$

Hence, (4.26) becomes

$$
\frac{d}{d t} \int_{\mathbb{R}^{2}} \varphi d\left(\bar{\mu}_{1}\right)_{\tau}(t)=\sum_{k=0}^{+\infty}\left(-\tau \int_{\mathbb{R}^{2}}\left\langle\nabla \varphi, \nabla h_{\mu_{\tau}^{k+1}}\right\rangle d\left(\mu_{1}\right)_{\tau}^{k+1}+\left(\mathbf{R}_{1}\right)_{\tau}^{k+1}\right) \delta_{\tau k}(t) .
$$

Reasoning similarly for $\left(\bar{\mu}_{2}\right)_{\tau}(t)$, we get

$$
\frac{d}{d t} \int_{\mathbb{R}^{2}} \varphi d\left(\bar{\mu}_{2}\right)_{\tau}(t)=\sum_{k=0}^{+\infty}\left(\tau \int_{\mathbb{R}^{2}}\left\langle\nabla \varphi, \nabla h_{\mu_{\tau}^{k+1}}\right\rangle d\left(\mu_{2}\right)_{\tau}^{k+1}+\left(\mathbf{R}_{2}\right)_{\tau}^{k+1}\right) \delta_{\tau k}(t) .
$$

From Theorem 4.1 and Proposition 4.1, we know that on a suitable subsequence, that we do not relabel, $\left(\bar{\mu}_{1}\right)_{\tau}(t)$ and $\left(\bar{\mu}_{2}\right)_{\tau}(t)$ converge weakly in $L^{p}\left(\mathbb{R}^{2}\right)$ to $\mu_{1}(t)$ and $\mu_{2}(t)$ respectively, for any $t$. Still by (4.11) and with Hölder inequality we find

$$
\begin{aligned}
\int_{0}^{+\infty} \mid \int_{\mathbb{R}^{2}} & \left.\left\langle\nabla \varphi, \nabla h_{\bar{\mu}_{\tau}(t)}\right\rangle d\left(\bar{\mu}_{1}\right)_{\tau}(t)\right|^{2} d t=\sum_{k=0}^{+\infty} \tau\left|\int_{\mathbb{R}^{2}}\left\langle\nabla \varphi, \nabla h_{\mu_{\tau}^{k+1}}\right\rangle d\left(\mu_{1}\right)_{\tau}^{k+1}\right|^{2} \\
& =\sum_{k=0}^{+\infty} \tau\left|\frac{1}{\tau} \int_{\mathbb{R}^{2} \times \mathbb{R}^{2}}\langle\nabla \varphi(x), x-y\rangle d\left(\gamma^{1}\right)_{\tau}^{k+1}(x, y)\right|^{2} \\
\leq & \sum_{k=0}^{+\infty} \frac{1}{\tau}\left(\sup _{\mathbb{R}^{2}}|\nabla \varphi|\right)^{2} \int_{\mathbb{R}^{2} \times \mathbb{R}^{2}}|x-y| d\left(\gamma^{1}\right)_{\tau}^{k+1}(x, y) \\
\leq & \alpha\left(\sup _{\mathbb{R}^{2}}|\nabla \varphi|\right)^{2} \sum_{k=0}^{+\infty} \frac{1}{\tau} W_{2}^{2}\left(\left(\mu_{1}\right)_{\tau}^{k},\left(\mu_{1}\right)_{\tau}^{k+1}\right) .
\end{aligned}
$$


The last term here is uniformly bounded in $\tau$, thanks to the basic estimate (4.25) of the discrete scheme. Therefore, we have obtained the weak $L^{2}(0,+\infty)$ compactness of the sequence

$$
\int_{\mathbb{R}^{2}}\left\langle\nabla \varphi, \nabla h_{\bar{\mu}_{\tau}(t)}\right\rangle d\left(\bar{\mu}_{1}\right)_{\tau}(t)
$$

Such sequence is also pointwise converging (for any $t$ ), to

$$
\int_{\mathbb{R}^{2}}\left\langle\nabla \varphi, \nabla h_{\mu(t)}\right\rangle d \mu_{1}(t)
$$

due to the weak convergence in $L^{p}\left(\mathbb{R}^{2}\right)$ of $\left(\bar{\mu}_{1}\right)_{\tau}(t)$ to $\mu_{1}(t)$ and the strong convergence in $L_{l o c}^{p}\left(\mathbb{R}^{2}\right)$ of $\nabla h_{\bar{\mu}_{\tau}(t)}$ to $\nabla h_{\mu(t)}$, coming from elliptic regularity. Then, as $\tau \rightarrow 0$ (and possibly passing to a further subsequence) the sequence in $(4.30)$ converges to $(4.31)$ in the weak $L^{2}(0,+\infty)$ topology as well. From this fact we deduce the convergence in the sense of distributions (in time)

$$
\sum_{k=0}^{+\infty} \tau \delta_{k \tau}(t) \int_{\mathbb{R}^{2}}\left\langle\nabla \varphi, \nabla h_{\mu_{\tau}^{k+1}}\right\rangle d\left(\mu_{1}\right)_{\tau}^{k+1} \rightarrow \int_{\mathbb{R}^{2}}\left\langle\nabla \varphi, \nabla h_{\mu(t)}\right\rangle d \mu_{1}(t),
$$

since for any $\psi \in C_{c}^{0}(0,+\infty)$ there holds

$$
\begin{aligned}
\int_{0}^{+\infty} \psi(t) & d\left(\sum_{k=0}^{+\infty} \tau \delta_{k \tau}(t) \int_{\mathbb{R}^{2}}\left\langle\nabla \varphi, \nabla h_{\mu_{\tau}^{k+1}}\right\rangle d\left(\mu_{1}\right)_{\tau}^{k+1}\right) \\
= & \int_{0}^{+\infty} \psi(\tau\lceil t / \tau\rceil) \int_{\mathbb{R}^{2}}\left\langle\nabla \varphi, \nabla h_{\bar{\mu}_{\tau}(t)}\right\rangle d\left(\bar{\mu}_{1}\right)_{\tau}(t) d t .
\end{aligned}
$$

The same for the analogous term involving $\left(\mu_{2}\right)_{\tau}^{k+1}$. About the remainder term, it is readily seen from (4.27) that

$$
\left|\left(\mathbf{R}_{1}\right)_{\tau}^{k+1}\right| \leq \frac{1}{2}\left(\sup _{\mathbb{R}^{2}}\left|\nabla^{2} \varphi\right|\right) W_{2}^{2}\left(\left(\mu_{1}\right)_{\tau}^{k},\left(\mu_{1}\right)_{\tau}^{k+1}\right),
$$

so that by (4.25) we have $\sum_{k=0}^{+\infty}\left(\mathbf{R}_{1}\right)_{\tau}^{k+1}=o(1)$ as $\tau \rightarrow 0$, and the same holds for $\left(\mathbf{R}_{2}\right)_{\tau}^{k}$. These estimates on the remainders, (4.32) and its analogous for $\mu_{2}$ allow to pass to the limit in (4.28) and (4.29) as $\tau \rightarrow 0$ : we get, in the sense of distributions,

$$
\left\{\begin{array}{l}
\frac{d}{d t} \int_{\mathbb{R}^{2}} \varphi d \mu_{1}(t)=-\int_{\mathbb{R}^{2}}\left\langle\nabla \varphi(x), \nabla h_{\mu(t)}\right\rangle d \mu_{1}(t), \\
\frac{d}{d t} \int_{\mathbb{R}^{2}} \varphi d \mu_{2}(t)=\int_{\mathbb{R}^{2}}\left\langle\nabla \varphi(x), \nabla h_{\mu(t)}\right\rangle d \mu_{2}(t),
\end{array}\right.
$$

for any $\varphi \in C_{0}^{2}\left(\mathbb{R}^{2}\right)$. Hence, the couple $\left(\mu_{1}(t), \mu_{2}(t)\right)$, with $\mu(t)=\mu_{1}(t)-\mu_{2}(t)$, is a distributional solution to (1.1).

Let us conclude with the proof of the energy dissipation formula. Integrating the relations in (4.33) from $s$ to $t$, and subtracting them, one obtains

$$
\int_{\mathbb{R}^{2}} \varphi d \mu(t)-\int_{\mathbb{R}^{2}} \varphi d \mu(s)=-\int_{s}^{t} \int_{\mathbb{R}^{2}}\left\langle\nabla h_{\mu(r)}, \nabla \varphi\right\rangle d\left(\mu_{1}(r)+\mu_{2}(r)\right) d r .
$$


Let us sum the corresponding equalities with $\varphi=h_{\mu(t)}$ and $\varphi=h_{\mu(s)}$. These functions are not compactly supported but still the computation makes sense: indeed, in this case the integrals above are finite, since $\Phi$ stays bounded along the flow and we have uniform $L^{4}$ bounds on $\mu(t)$ and, from (4.12), uniform $L^{\infty}$ bounds on $\nabla h_{\mu(t)}$. Thanks to $(4.6)$ we obtain

$\Phi(\mu(t))-\Phi(\mu(s))=-\frac{1}{2} \int_{s}^{t} \int_{\mathbb{R}^{2}}\left\langle\nabla h_{\mu(r)}, \nabla h_{\mu(t)}+\nabla h_{\mu(s)}\right\rangle d\left(\mu_{1}(r)+\mu_{2}(r)\right) d r$.

This relation, holding for any $0 \leq s \leq t$, yields absolute continuity for $t \mapsto$ $\Phi(\mu(t))$ and the desired dissipation equality (1.4).

\section{Uniqueness via Lagrangian approach}

In order to prove uniqueness, let $T>0$ be small enough and let us consider two solutions

$$
\begin{array}{r}
\left(\mu_{1}, \mu_{2}\right) \in\left(\mathcal{M}_{\alpha}^{2}(\bar{\Omega}) \times \mathcal{M}_{\beta}^{2}(\bar{\Omega})\right) \cap L^{\infty}\left((0, T) ; L^{\infty}(\Omega)^{2}\right), \\
\left(\nu_{1}, \nu_{2}\right) \in\left(\mathcal{M}_{\alpha}^{2}(\bar{\Omega}) \times \mathcal{M}_{\beta}^{2}(\bar{\Omega})\right) \cap L^{\infty}\left((0, T) ; L^{\infty}(\Omega)^{2}\right)
\end{array}
$$

to (1.1)-(1.2), starting from the $L^{\infty}(\Omega)^{2}$ initial data $\left(\mu_{1}^{0}, \mu_{2}^{0}\right)$ and $\left(\nu_{1}^{0}, \nu_{2}^{0}\right)$ respectively, where $\mu_{1}^{0}, \mu_{2}^{0}, \nu_{1}^{0}, \nu_{2}^{0}$ are compactly supported in $\Omega$. These solutions do not give mass to the boundary. As usual, we will let $\mu(t)=\mu_{1}(t)-\mu_{2}(t)$ and $\nu(t)=\nu_{1}(t)-\nu_{2}(t)$. The following is the proof of the main uniqueness result. It is global in time in the case $\Omega=\mathbb{R}^{2}$. Otherwise we have a short time result (that is $T$ is such that no mass goes on the boundary for $t<T$ ).

Remark 5.1. The global uniqueness in time of $L^{\infty}$ solutions in presence of boundary (and such that no mass reenters in the interior) has been proven in [17] for the single equation of (1.1). Here we present a Lagrangian approach which makes use of a $H^{-1}$-Wasserstein estimate as (5.7) below (see also [15], where the same technique is exploited for the study of the Vlasov-Poisson equation). Notice that (5.7) fails as soon as some mass reaches the boundary.

\section{Proof of Theorem 1.2}

Let $\mu_{1}(t), \mu_{2}(t), \nu_{1}(t), \nu_{2}(t)$ be as above. Existence of such $L^{\infty}$ solutions comes from Theorem 1.1 and from the following fact: if the initial data are compactly supported, there exists $T>0$ such that no mass reaches the boundary before $t=T$. Indeed, we have a finite speed of propagation property for the masses involved, since the velocity vector field $\pm \nabla h_{\mu(t)}$ is uniformly bounded, as remarked in the previous section, as soon as we have uniform $L^{p}$ bounds $(p \geq 4)$ on $\mu(t)$. Therefore, we have solutions which really stay in $L^{\infty}(\Omega)$ for $t \in(0, T)$, without leaking of mass to the boundary.

We are going to show that, if $\mu_{1}^{0}=\nu_{1}^{0}$ and $\mu_{2}^{0}=\nu_{2}^{0}$, then $\mu_{1}(t)=\nu_{1}(t)$ and $\mu_{2}(t)=\nu_{2}(t)$ for all $t \in(0, T)$. Let $U_{1}^{0}:=\mu_{1}^{0}=\nu_{1}^{0}$ and $U_{2}^{0}:=\mu_{2}^{0}=\nu_{2}^{0}$. With the lagrangian point of view, we consider the flows associated to the velocity vector fields of the two equations of (1.1), in correspondence of $\mu$ and $\nu$. They 
are defined by

$$
\begin{array}{ll}
\dot{X}_{\mu}=-\nabla h_{\mu}\left(t, X_{\mu}\right), & \dot{X}_{\nu}=-\nabla h_{\nu}\left(t, X_{\nu}\right), \\
\dot{Y}_{\mu}=\nabla h_{\mu}\left(t, Y_{\mu}\right), & \dot{Y}_{\nu}=\nabla h_{\nu}\left(t, Y_{\nu}\right) .
\end{array}
$$

We recall a log-Lipschitz property, satisfied by $\nabla h_{\rho}$ when $\rho \in L^{\infty}(\Omega) \cap$ $\mathcal{M}_{\alpha}^{2}(\bar{\Omega})$ (see for instance [18]). For any $t \in[0, T)$ and for small enough $|x-y|$ there holds

$$
\left|\nabla h_{\rho}(t, x)-\nabla h_{\rho}(t, y)\right| \leq C|x-y||\log | x-y||,
$$

where $C$ is a constant depending on $\|\rho\|_{L^{\infty}(\Omega)}$. The log-Lipschitz regularity ensures the continuity (in both variables) of the flows defined in (5.1), see [18].

Then we write the solutions $\mu_{1}, \nu_{1}, \mu_{2}, \nu_{2}$ as

$$
\begin{array}{ll}
\mu_{1}=\left(X_{\mu}\right)_{\#} U_{1}^{0}, & \nu_{1}=\left(X_{\nu}\right)_{\#} U_{1}^{0}, \\
\mu_{2}=\left(Y_{\mu}\right)_{\#} U_{2}^{0}, & \nu_{2}=\left(Y_{\nu}\right)_{\#} U_{2}^{0} .
\end{array}
$$

Let us define

$$
\begin{aligned}
\Psi_{X}(t) & :=\int_{\Omega}\left|X_{\mu}(t, x)-X_{\nu}(t, x)\right|^{2} U_{1}^{0}(x) d x, \\
\Psi_{Y}(t) & :=\int_{\Omega}\left|Y_{\mu}(t, x)-Y_{\nu}(t, x)\right|^{2} U_{2}^{0}(x) d x .
\end{aligned}
$$

Making use of (5.3) we have

$$
\begin{aligned}
W_{2}^{2}\left(\mu_{1}(t), \nu_{1}(t)\right) & \leq \int_{\Omega \times \Omega}|x-y|^{2} d\left[\left(X_{\mu}(t, \cdot), X_{\nu}(t, \cdot)\right)_{\#} U_{1}^{0}\right](x, y) \\
& =\int_{\Omega}\left|X_{\mu}(t, x)-X_{\nu}(t, x)\right|^{2} d U_{1}^{0}(x)=\Psi_{X}(t),
\end{aligned}
$$

and analogously for $W_{2}^{2}\left(\mu_{2}(t), \nu_{2}(t)\right)$, so that

$$
W_{2}^{2}\left(\mu_{1}(t), \nu_{1}(t)\right)+W_{2}^{2}\left(\mu_{2}(t), \nu_{2}(t)\right) \leq \Psi_{X}(t)+\Psi_{Y}(t) .
$$

Now we estimate the derivative of $\Psi_{X}$. Consider that

$$
\begin{aligned}
& \frac{d}{d t} \Psi_{X}(t) \\
& =2 \int_{\Omega} U_{1}^{0}(x)\left\langle\left(X_{\mu}(t, x)-X_{\nu}(t, x)\right),\left(-\nabla h_{\mu}\left(t, X_{\mu}(t, x)\right)+\nabla h_{\nu}\left(t, X_{\nu}(t, x)\right)\right)\right\rangle \\
& \leq 2 \int_{\Omega} U_{1}^{0}(x)\left|X_{\mu}(t, x)-X_{\nu}(t, x)\right|\left|\nabla h_{\mu}\left(t, X_{\mu}(t, x)\right)-\nabla h_{\nu}\left(t, X_{\nu}(t, x)\right)\right| \\
& \leq 2 \int_{\Omega} U_{1}^{0}(x)\left|X_{\mu}(t, x)-X_{\nu}(t, x)\right|\left|\nabla h_{\mu}\left(t, X_{\mu}(t, x)\right)-\nabla h_{\nu}\left(t, X_{\mu}(t, x)\right)\right| \\
& \quad+2 \int_{\Omega} U_{1}^{0}(x)\left|X_{\mu}(t, x)-X_{\nu}(t, x)\right|\left|\nabla h_{\nu}\left(t, X_{\mu}(t, x)\right)-\nabla h_{\nu}\left(t, X_{\nu}(t, x)\right)\right| .
\end{aligned}
$$


By means of the Cauchy-Schwarz inequality we have

$$
\begin{aligned}
& \frac{d}{d t} \Psi_{X}(t) \\
& \leq 2\left(\Psi_{X}(t)\right)^{1 / 2}\left(\int_{\Omega} U_{1}^{0}(x)\left|\nabla h_{\mu}\left(t, X_{\mu}(t, x)\right)-\nabla h_{\nu}\left(t, X_{\mu}(t, x)\right)\right|^{2} d x\right)^{1 / 2} \\
& \quad+2\left(\Psi_{X}(t)\right)^{1 / 2}\left(\int_{\Omega} U_{1}^{0}(x)\left|\nabla h_{\nu}\left(t, X_{\mu}(t, x)\right)-\nabla h_{\nu}\left(t, X_{\nu}(t, x)\right)\right|^{2} d x\right)^{1 / 2} .
\end{aligned}
$$

We introduce the further notation

$$
\begin{aligned}
\Theta(t) & :=\int_{\Omega} U_{1}^{0}(x)\left|\nabla h_{\mu}\left(t, X_{\mu}(t, x)\right)-\nabla h_{\nu}\left(t, X_{\mu}(t, x)\right)\right|^{2} d x, \\
\Lambda(t) & :=\int_{\Omega} U_{1}^{0}(x)\left|\nabla h_{\nu}\left(t, X_{\mu}(t, x)\right)-\nabla h_{\nu}\left(t, X_{\nu}(t, x)\right)\right|^{2} d x .
\end{aligned}
$$

Concerning $\Theta$ we have

$$
\begin{aligned}
\Theta(t) & =\int_{\Omega}\left|\nabla h_{\mu}(t, x)-\nabla h_{\nu}(t, x)\right|^{2} d\left[X_{\mu}(t, \cdot)_{\#} U_{1}^{0}\right](x) \\
& =\int_{\Omega} \mu_{1}(t, x)\left|\nabla h_{\mu}(t, x)-\nabla h_{\nu}(t, x)\right|^{2} d x \\
& \leq\left\|\mu_{1}(t, \cdot)\right\|_{L^{\infty}(\Omega)}\left\|\nabla h_{\mu}(t, \cdot)-\nabla h_{\nu}(t, \cdot)\right\|_{L^{2}(\Omega)}^{2} .
\end{aligned}
$$

Next we make use of the equivalence of the norms $\left\|\nabla h_{\rho}\right\|_{L^{2}(\Omega)}$ and $\|\rho\|_{H^{-1}(\Omega)}$. Moreover, we take advantage of the inequality

$$
\left\|\rho_{1}-\rho_{2}\right\|_{H^{-1}(\Omega)} \leq \max \left\{\left\|\rho_{1}\right\|_{L^{\infty}(\Omega)}^{1 / 2},\left\|\rho_{2}\right\|_{L^{\infty}(\Omega)}^{1 / 2}\right\} W_{2}\left(\rho_{1}, \rho_{2}\right),
$$

holding for any couple of $L^{\infty}$ probability densities on $\Omega$, as shown in $[15$, Proposition 2.8]. Such result naturally extends to densities of positive measures with same total mass.

Thus from (5.6) we get

$$
\begin{aligned}
\Theta(t) & \leq\left\|\mu_{1}\right\|_{L^{\infty}(\Omega)}\left\|\nabla h_{\mu}-\nabla h_{\nu}\right\|_{L^{2}(\Omega)}^{2} \\
& \leq K\left\|U_{1}^{0}\right\|_{L^{\infty}(\Omega)}\left\|\left(\mu_{1}(t, \cdot)-\mu_{2}(t, \cdot)\right)-\left(\nu_{1}(t, \cdot)-\nu_{2}(t, \cdot)\right)\right\|_{H^{-1}(\Omega)}^{2} \\
& \leq 2 K\left\|U_{1}^{0}\right\|_{L^{\infty}(\Omega)}\left(\left\|\mu_{1}(t, \cdot)-\nu_{1}(t, \cdot)\right\|_{H^{-1}(\Omega)}^{2}+\left\|\mu_{2}(t, \cdot)-\nu_{2}(t, \cdot)\right\|_{H^{-1}(\Omega)}^{2}\right) \\
& \leq 2 K\left\|U_{1}^{0}\right\|_{L^{\infty}(\Omega)}^{2}\left(W_{2}^{2}\left(\mu_{1}(t), \nu_{1}(t)\right)+W_{2}^{2}\left(\mu_{2}(t), \nu_{2}(t)\right)\right) \\
& \leq 2 K\left\|U_{1}^{0}\right\|_{L^{\infty}(\Omega)}^{2}\left(\Psi_{X}(t)+\Psi_{Y}(t)\right),
\end{aligned}
$$

where the last inequality follows from (5.4).

In order to find an estimate for $\Lambda$, we take advantage of the log-Lipschitz inequality (5.2), satisfied by $\nabla h_{\mu}$ and $\nabla h_{\nu}$. Choosing $T$ small enough such that the quantity

$$
\left\|X_{\mu}(\cdot, \cdot)-X_{\nu}(\cdot, \cdot)\right\|_{L^{\infty}((0, T) \times \Omega)}
$$


is small (this is possible since $X_{\mu}, X_{\nu}$ are uniformly continuous), we have, for $t<T$,

$$
\begin{aligned}
\Lambda(t) & \leq C^{2} \int_{\Omega} U_{1}^{0}(x)\left|X_{\mu}(t, x)-X_{\nu}(t, x)\right|^{2} \log ^{2}\left|X_{\mu}(t, x)-X_{\nu}(t, x)\right| d x \\
& \leq \frac{C^{2}}{4} \int_{\Omega} U_{1}^{0}(x)\left|X_{\mu}(t, x)-X_{\nu}(t, x)\right|^{2} \log ^{2}\left|X_{\mu}(t, x)-X_{\nu}(t, x)\right|^{2} d x \\
& \leq \frac{C^{2}}{4} \Psi_{X}(t) \log ^{2} \Psi_{X}(t),
\end{aligned}
$$

where we made use of the Jensen inequality.

Inserting the estimates for $\Theta$ and $\Lambda$ in (5.5) we obtain

$$
\frac{d}{d t} \Psi_{X}(t) \leq 2 \sqrt{K}\left\|U_{1}^{0}\right\|_{L^{\infty}(\Omega)}^{2} \sqrt{\Psi_{X}^{2}(t)+\Psi_{X}(t) \Psi_{Y}(t)}+C \Psi_{X}(t)\left|\log \Psi_{X}(t)\right| .
$$

The estimate for the derivative of $\Psi_{Y}$ is the very same, so that

$$
\frac{d}{d t} \Psi_{Y}(t) \leq 2 \sqrt{K}\left\|U_{2}^{0}\right\|_{L^{\infty}(\Omega)}^{2} \sqrt{\Psi_{Y}^{2}(t)+\Psi_{Y}(t) \Psi_{X}(t)}+C \Psi_{Y}(t)\left|\log \Psi_{Y}(t)\right| .
$$

Moreover we can find a positive constant $M$ such that

$$
\frac{d}{d t} \Psi_{X}(t) \leq M\left[\Psi_{X}(t)+\Psi_{Y}(t)+\Psi_{X}(t)\left|\log \Psi_{X}(t)\right|\right] .
$$

Since the map $x \mapsto x|\log x|$ is increasing for small $x$, and since $\Psi_{X}, \Psi_{Y}$ are small for small $T$ (they are continuous in $t$ ), we can also infer, omitting for simplicity the dependence on $t$,

$$
\dot{\Psi}_{X} \leq M\left[\Psi_{X}+\Psi_{Y}+\left(\Psi_{X}+\Psi_{Y}\right)\left|\log \left(\Psi_{X}+\Psi_{Y}\right)\right|\right] .
$$

Symmetrically for $\Psi_{Y}$, there exist a positive constant $M^{\prime}$ such that, for small enough $T$,

$$
\dot{\Psi}_{Y} \leq M^{\prime}\left[\Psi_{Y}+\Psi_{X}+\left(\Psi_{Y}+\Psi_{X}\right)\left|\log \left(\Psi_{Y}+\Psi_{X}\right)\right|\right]
$$

Summing the last two inequalities we get

$$
\dot{\Psi}_{X}+\dot{\Psi}_{Y} \leq \max \left\{M, M^{\prime}\right\}\left[\Psi_{X}+\Psi_{Y}+\left(\Psi_{X}+\Psi_{Y}\right)\left|\log \left(\Psi_{X}+\Psi_{Y}\right)\right|\right] .
$$

Since $\int_{0}^{\varepsilon} \frac{d x}{x|\log x|}=\infty$ for any $\varepsilon>0$, the standard Osgood condition is satisfied and the differential inequality admits only the zero solution: we conclude that $\Psi_{X}(t)=\Psi_{Y}(t)=0$. Then we have from (5.4)

$$
W_{2}^{2}\left(\mu_{1}(t), \nu_{1}(t)\right)+W_{2}^{2}\left(\mu_{2}(t), \nu_{2}(t)\right) \leq 0,
$$

so that $\mu_{1}(t)=\nu_{1}(t)$ and $\mu_{2}(t)=\nu_{2}(t)$.

\section{Acknowledgments}

The author acknowledges the support from the project FP7-IDEAS-ERC-StG Grant \#200497 (BioSMA). For many fruitful discussions, he also wishes to thank Luigi Ambrosio, Giovanni De Matteis, Sylvia Serfaty, Ulisse Stefanelli and Epifanio Virga. 


\section{References}

[1] Ambrosio, L., Gigli, N., Savaré, G.: Gradient flows in metric spaces and in the space of probability measures. Lectures in Mathematics ETH Zürich, Birkhäuser Verlag, Basel (2005)

[2] Ambrosio, L., Mainini, E., Serfaty, S.: Gradient flow of the ChapmanRubinstein-Schatzman model for signed vortices. Ann. Inst. H. Poincaré Anal. Non Linéaire 28(2), 217-246 (2011)

[3] Ambrosio, L., Serfaty, S.: A gradient flow approach to an evolution problem arising in superconductivity. Commun. Pure Appl. Math. LXI 11, 1495-1539 (2008)

[4] Brenier, Y.: Polar factorization and monotone rearrangement of vector-valued functions. Commun. Pure Appl. Math. 44, 375-417 (1991)

[5] Chapman, J.S.: A mean-field model of superconducting vortices in three dimensions. SIAM J. Appl. Math. 55(5), 1259-1274 (1995)

[6] Chapman, S.J., Rubinstein, J., Schatzman, M.: A mean-field model for superconducting vortices. Eur. J. Appl. Math. 7(2), 97-111 (1996)

[7] DiPerna, R.J., Lions, P.L.: Ordinary differential equations, transport theory and Sobolev spaces. Invent. Math. 98, 511-547 (1989)

[8] Du, Q., Zhang, P.: Existence of weak solutions to some vortex density models. SIAM J. Math. Anal. 34, 1279-1299 (2003)

[9] E, W.: Dynamics of vortices in Ginzburg-Landau theories with applications to superconductivity. Phys. D 77, 383-404 (1994)

[10] E, W.: Dynamics of vortex-liquids in Ginzburg-Landau theories with applications to superconductivity. Phys. Rev. B. 50(3), 1126-1135 (1994)

[11] Elliott, C.M., Styles, V.: Numerical analysis of a mean field model of superconducting vortices. IMA J. Numer. Anal. 21, 1-51 (2001)

[12] Evans, L.C.: Partial differential equations. In: Graduate Studies in Mathematics, vol. 19. American Mathematical Society, Providence (1998)

[13] Gilbarg, D., Trudinger, N.: Elliptic partial differential equations of second order, 3rd edn. Springer, Berlin (1998)

[14] Jordan, R., Kinderlehrer, D., Otto, F.: The variational formulation of the Fokker-Planck equation. SIAM J. Math. Anal. 29, 1-17 (1998)

[15] Loeper, G.: Uniqueness of the solution to the Vlasov-Poisson system with bounded densitiy. J. Math. Pures Appl. 86, 68-79 (2006)

[16] Lin, F.H., Zhang, P.: On the hydrodynamic limit of Ginzburg-Landau vortices. Discrete Cont. Dyn. Syst. 6, 121-142 (2000) 
[17] Mainini, E.: A global uniqueness result for an evolution problem arising in superconductivity. Boll. Unione Mat. Ital. (9) II(2), 509-528 (2009)

[18] Majda, A.J., Bertozzi, A.L.: Vorticity and incompressible flow. Cambridge Texts of Applied Mathematics, vol. 27. Cambridge University Press, Cambridge (2002)

[19] McCann, R.: A convexity principle for interacting gases. Adv. Math. 128, 153-179 (1997)

[20] Masmoudi, N., Zhang, P.: Global solutions to vortex density equations arising from sup-conductivity. Ann. Inst. H. Poincaré Anal. Non Linéaire 22(4), 441-458 (2005)

[21] Otto, F.: Dynamics of labyrinthine pattern formation in magnetic fluids: a meanfield theory. Arch. Ration. Mech. Anal. 141, 63-103 (1998)

[22] Serfaty, S.: On a model of rotating superfluids. ESAIM Control Opt. Calcul Des Variat. 6, 201-238 (2001)

[23] Sandier, E., Serfaty, S.: A rigorous derivation of a free-boundary problem arising in superconductivity. Ann. Sci. Ecole Norm. Sup. 4E Ser. 33, 561-592 (2000)

[24] Schaetzle, R., Styles, V.: Analysis of a mean field model of superconducting vortices. Eur. J. Appl. Math. 10, 319-352 (1999)

[25] Villani, C.: Topics in optimal transportation. In: Graduate Studies in Mathematics, vol. 58. American Mathematical Society, Providence (2003)

[26] Villani, C.: Optimal transport, old and new. Springer, Berlin (2008)

[27] Yudovich, V.: Nonstationary flow of an ideal incompressible liquid. Zhurn. Vych. Mat. 3, 1032-1066 (1963)

Edoardo Mainini

Dipartimento di Matematica 'F. Casorati'

Università degli Studi di Pavia

via Ferrata 1

27100 Pavia, Italy

e-mail: edoardo.mainini@unipv.it

Received: 7 September 2010.

Revised: 23 May 2011.

Accepted: 24 May 2011. 